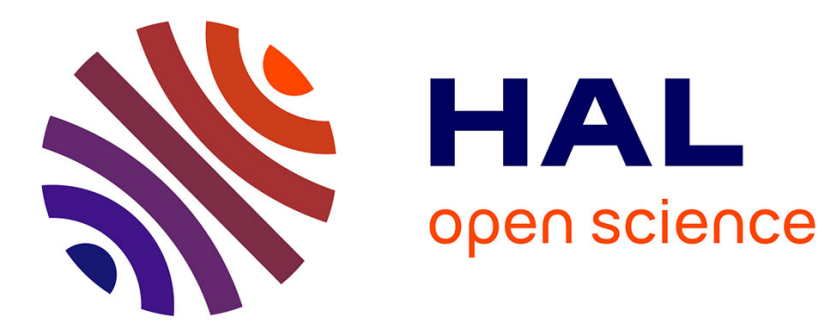

\title{
Option pricing with discrete time jump processes
}

Dominique Guegan, Florian Ielpo, Hanjarivo Lalaharison

\section{To cite this version:}

Dominique Guegan, Florian Ielpo, Hanjarivo Lalaharison. Option pricing with discrete time jump processes. 2012. halshs-00611706v2

\section{HAL Id: halshs-00611706 \\ https://shs.hal.science/halshs-00611706v2}

Submitted on 17 Jul 2012

HAL is a multi-disciplinary open access archive for the deposit and dissemination of scientific research documents, whether they are published or not. The documents may come from teaching and research institutions in France or abroad, or from public or private research centers.
L'archive ouverte pluridisciplinaire HAL, est destinée au dépôt et à la diffusion de documents scientifiques de niveau recherche, publiés ou non, émanant des établissements d'enseignement et de recherche français ou étrangers, des laboratoires publics ou privés. 


\section{Documents de Travail du Centre d'Economie de la Sorbonne}
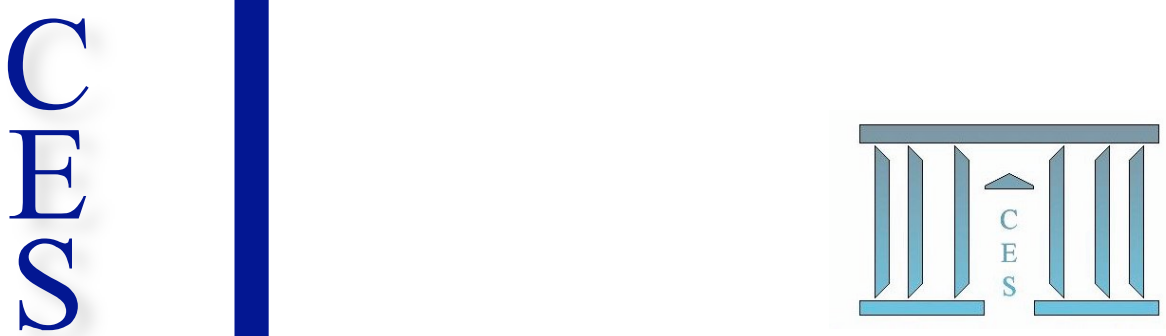

Option Pricing with Discrete Time Jump Processes

Dominique GUEGAN, Florian IELPO, Hanjarivo LALAHARISON

2011.37

Version révisée

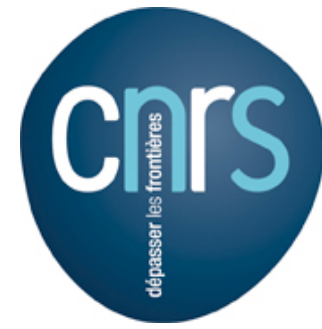




\title{
Option Pricing with Discrete Time Jump Processes
}

\author{
Dominique Guégan* Florian Ielpo ${ }^{\dagger}$ Hanjarivo Lalaharison ${ }^{\ddagger}$
}

April 17, 2012

\begin{abstract}
In this paper we propose new option pricing models based on class of models with jump contain in the Lévy-type based models (NIG-Lévy, Merton-jump (Merton 1976) and Duan based model (Duan 2007)). By combining these different class of models with several volatility dynamics of the GARCH type, we aim at taking into account the dynamics of financial returns in a realistic way. The associated risk neutral dynamics of the time series models is obtained through two different specifications for the pricing kernel: we provide a characterization of the change in the probability measure using the Esscher transform and the Minimal Entropy Martingale Measure. We finally assess empirically the performance of this modelling approach, using a dataset of European options based on the S\&P 500 and on the CAC 40 indices. Our results show that models involving jumps and a time varying volatility provide realistic pricing results for options with different kinds of time to maturities and moneyness. Furthermore, our results provide evidence of consistency between historical and risk neutral distributions, making the approach developed here interesting to price option when option markets are illiquid or when such markets simply do not exist.
\end{abstract}

Keywords: Option pricing, Lévy processes, exponential affine Stochastic Discount Factor, Minimal Entropy Martingale Measure, CAC 40, S\&P 500.

\footnotetext{
${ }^{*}$ University Paris1 Panthéon - Sorbonne, CES UMR 8174, 106 bd de l'Hôpital, 75013 Paris, France. Email: dguegan@univ-paris1.fr. Tel: +33140078298.

${ }^{\dagger}$ BCV Asset Management, Place St François 14, P.O. Box 300, 10001 Lausanne, switzerland. Email: florian.ielpo@bcv.ch. Tel: +4121212 2035.

${ }^{\ddagger}$ University Paris 1 Panthéon-Sorbonne, MSE, 106 bd de l’Hôpital, 75013 Paris, France. Email: lalaharisonh@gmail.com.
} 


\section{Introduction}

When the concept of volatility aims at measuring the state of risks at work in financial markets, jumps offer a unique opportunity to represent the fundamental process through which market quotes integrate the relevant information flow over trading days. The inspection of both daily and intraday data sets reveals that the jump activity implied by the returns on various assets should not be ignored: the returns are characterized by tails whose thickness cannot be accounted for through any conditionally Gaussian model, and whose fit is clearly improved through the use of jumps. Intraday data based tests improved our understanding over both the size and the intensity of the jump processes, unveiling how complex the blending of jumps and volatility can be. These considerations have led to the idea that a valuation model for financial assets should include both these components. This article proposes an empirical discrete time Lévy-process approach to the pricing of European options on equity indexes. We discuss the key ingredients to be considered to achieve this objective and we empirically circumvent the usual pitfalls of such an approach, as the non-uniqueness of the pricing measure and the choice of a pricing kernel specification. One of the key aspect of the proposed pricing method is that option prices are obtained using the historical measure $\mathbb{P}$ and discounting the terminal payoff with the stochastic discount factor. It does not require liquid option prices to calibrate the risk neutral $\mathbb{Q}$.

The introduction of jumps into financial models present various advantages, either in terms of time series analysis or in terms of option pricing. The deviation of historical returns from the Black Scholes (1973) hypothesis of Gaussianity is now well known and documented, see for example Eberlein and Prause (2002), Bouchaud and Potters (2003) or Embrecht et al. (2005). It led to the development of various time series models usually combining a GARCH component with a non-Gaussian distribution or a jump process. The use of Lévy processes is by no means specific to time series analysis: various continuous time finance models are built on the combination of a process of the Heston (1993) type for the conditional variance with a jump component, either in the dynamics of the variance or of the log-returns. Examples of this sort are available in Merton (1973), Bates (1996) or Duffie et al. (2000). When the obvious interest of jumps in time series analysis lies within the fit of the tails of returns' distribution, the incorporation of jumps into a continuous time finance option pricing model is essential to the pricing of short term options: the convexity of the implied volatility smile is sharper for such options and stochastic volatility models are unable to replicate this stylized fact without a jump component. On this point, we refer to Duffie et al. (2000).

Even though continuous time finance provided market participants with a convenient and fast way to compute prices for various contingent claims, it offers a framework that can hardly handle the key issue of bringing together the historical and the risk neutral distributions. If the calibration of parameters using option prices is a relatively straightforward exercise, the estimation of the parameters solely using the historical time series of returns is often tricky: some references are for example the methodologies that are used in Eracker et al. (2003), Chacko and Viceira (2003) or Michael and Maria 
(2005). Option pricing in a discrete time framework has over the years proved to be a fruitful and economically appealing means to understand how the time series of returns relate to the price of hedging vehicles such as European options, as presented for example in Rosenberg et al. (2002), Brown and Jackwerth (2001), Barone-Adesi et al. (2008), Chorro et al. (2010a,2012) among others. Instead of putting the emphasize on obtaining closed form expressions for option pricing formulas as for most of the continuous time literature, this stream of literature rather focuses on turning time series models that are consistent with the behaviour of returns into option prices. This kind of approach is all the more important as option prices are not always reliable, as it has been the case during the liquidity squeeze of 2008. Finally, this research direction is of a primary importance to compute the price of derivatives for which there is no existing market: for such cases, the option pricing model's parameters have to be estimated from the asset's returns and time series model are designed for such purposes.

In this article, we present and compare various option pricing models based on Lévy processes. Each of these models combine different volatility structures, conditional distributions and pricing kernels, the link between each of them being the use of an unconditional distribution which characterises a Lévy process. We define the change in the probability measure based on various assumptions regarding the pricing kernel. The volatility models used here are the EGARCH (Nelson, 1991), the GARCH-GJR (Glosten et al, 1993) and the APARCH model ${ }^{1}$ (Ding et al, 1993). Three Lévy process specifications are considered under the historical distribution. First, we introduce a structural Lévy model whose particular model is the Normal Inverse Gaussian model. Second, we consider the compound Poisson model proposed by Merton (1976). Third, we consider the compound Poisson model by Duan et al. (2007). These processes are driven by a finite number of jumps within a finite time interval.

Appealing though the discrete time approach may be, it nonetheless requires coping with an additional issue: as explained in Chorro et al. (2010a), with such an approach, the risk neutral distribution is no longer unique and a choice must be made regarding the shape of the market's aggregated risk aversion implied by the selected pricing kernel. We focus on two representations for the pricing kernel: the Esscher transform (ESS) martingale measure which corresponds to an exponential affine stochastic discount factor and the Minimal Entropy Martingale Measure (MEMM) (or extended Girsanov principle) (Elliot and Madan, 1998). The equivalence between these two approaches has been proved inside a Gaussian framework (Badescu and Kulberger, 2007), but such equivalence is not available outside this framework. We prove the stability of the conditional distribution under the historical and risk neutral distribution and we exhibit the required change in the parameters of the historical distribution to obtain the risk neutral one. One novelty of our article is to compute the expression under the

\footnotetext{
${ }^{1}$ The selected GARCH models differ from the very specific GARCH representation used in Christoffersen et al. (2006) that relates to the Inverse Gaussian distribution used in their model. The combination of the two enables the authors to obtain a close form expression for the characteristic function of the return under the risk neutral distribution. Our approach in this paper is different: we do not seek at computing close form expression, but rather at using empirically grounded models and test their ability to deliver option prices close what is observed on financial markets.
} 
pricing measure of the pricing formula when we use GARCH-type processes with Lévy innovations, both using the ESS and MEMM approaches. We also provide the expression of the pricing distribution for a GARCH-type model with Poisson jumps using the Esscher transform. In the case of the MEMM approach, the result has already been established by Fujiwara and Miyahara (2003).

Finally, we assess the quality of the modelling approaches selected here using a dataset of European options on the S\&P 500 and on the CAC 40 indices. Our empirical results point in several directions: first, this type of approach combining an empirical strategy with discrete time models provides minimum pricing errors that are broadly consistent with several articles based on a direct calibration of the models' parameters to option prices. We also find that a model based on an EGARCH volatility specification combined with a simple jump process generally provides us with the lowest mispricing errors. More importantly, our results indicate that the choice of the pricing kernel has a limited impact on the pricing errors, suggesting that the main driver of these errors is rather the underlying time series model rather than the specification of the link between the historical and the risk neutral distributions. Again, this is an essential message for this time series option pricing approach and its future perspectives.

This article is organized as follows. Section 2 presents the modelling approach retained here, along with the characterization of the risk neutral distribution associated with each model. Section 3 presents the empirical results and Section 4 concludes. Proofs are presented in the appendix.

\section{A Lévy-Based Discrete Time Modelling Approach}

This Section presents the Lévy-based time series models this article is based on. We discuss the model properties and then develop the change in probability measures required to obtain the risk neutral distribution essential to option pricing.

\subsection{Lévy-based time series models for financial returns}

Our approach combines different time varying volatility dynamics with Lévy processes. The models considered here are based on three different ways of dealing with jumps:

1. For the first specification, jumps affect both the returns $\left(Y_{t}\right)_{t}$ and the volatility $\left(h_{t}\right)_{t}$ :

$$
Y_{t}=\log \left(\frac{S_{t}}{S_{t-1}}\right)=\widetilde{m}_{t}+\sqrt{h_{t}} z_{t}, \quad S_{0}=s,
$$

where $\tilde{m}_{t}=r+m_{t},\left(h_{t}\right)_{t}$ is the conditional volatility obtained from any type of GARCH model. $\left(z_{t}\right)_{t}$ is a Lévy process with triplet $\left(c, \sigma^{2}, U\right)$, (Schoutens, 2003), 
$c \in \mathbb{R}, \sigma^{2} \geq 0$ and $U$ is the positive Lévy measure defined on $\mathbb{R} \backslash\{0\}$ satifying

$$
\int_{\mathbb{R}} \inf \left\{1, z^{2}\right\} U(d z)<\infty
$$

Inside these models we assume that the innovations $\left(z_{t}\right)_{t}$ follow a NIG-Lévy process which has no Brownian component and whose Lévy triplet is given by $(c, 0, U)$ with

$$
c=\mu+\frac{2 \delta \alpha}{\pi} \int_{0}^{1} \sinh (\beta z) K_{1}(\alpha z) d z
$$

$(\alpha, \beta, \delta, \mu) \in \mathbb{R}^{4}$ with $\delta>0$ and $0<|\beta|<\alpha, K_{1}$ is the modified Bessel function of the third kind, and the Lévy measure which determines the jump behavior of discontinuous Lévy processes is:

$$
U(d z)=u(z) d z=\frac{\delta \alpha}{\pi|z|} \exp (\beta z) K_{1}(\alpha|z|) d z .
$$

2. In the second specification, jumps affect only the returns $\left(Y_{t}\right)_{t}$ :

$$
Y_{t}=\log \left(\frac{S_{t}}{S_{t-1}}\right)=\tilde{m}_{t}+\sqrt{h_{t}} z_{t}+\sum_{j=1}^{N_{t}} V_{j}, \quad S_{0}=s
$$

where the innovations $z_{t}$ are characterized by a standard normal distribution, $N_{t}$ is a Poisson process with intensity $\lambda$, and $V_{1}, V_{2}, \ldots$ is a sequence of identically independent distributed random variables $N\left(\mu, \sigma^{2}\right)$, independent of the Poisson Process $N_{t}$.

3. In the third specification, jumps affect both the volatility and the returns:

$$
Y_{t}=\log \left(\frac{S_{t}}{S_{t-1}}\right)=\widetilde{m}_{t}+\sqrt{h_{t}}\left(z_{t}+\sum_{j=1}^{N_{t}} V_{j}\right), \quad S_{0}=s,
$$

where the innovations $z_{t}$ are characterized by a standard normal distribution, $N_{t}$ is a Poisson process with intensity $\lambda$, and $V_{1}, V_{2}, \ldots$ is a sequence of identically independent distributed random variables $N\left(\mu, \sigma^{2}\right)$, independent of the Poisson Process $N_{t}$.

In all models,

$$
\left\{\begin{array}{l}
m_{t}=\lambda_{0} \sqrt{h_{t}}-\frac{1}{2} h_{t} ; \quad \lambda_{0} \in \mathbb{R}, \\
h_{t}=G\left(h_{t-1}, Y_{t-1}\right) \text { is a related GARCH model. }
\end{array}\right.
$$

$\lambda_{0}$ is the constant unit risk premium. The conditional volatility is modelled by different GARCH models. Denoting $\varepsilon_{t}=\sqrt{h_{t}} z_{t}$, we use:

- the EGARCH(1,1) modelling (Nelson, 1991)

$$
\log \left(h_{t+1}\right)=\alpha_{0}+\beta_{1} \log \left(h_{t}\right)+\alpha_{1}\left(\left|z_{t}\right|-E\left[\left|z_{t}\right|\right]\right)+\gamma_{1} z_{t},
$$


- the GARCH-GJR(1,1) model introduced by Glosten, Jagannathan and Runkle (1993)

$$
h_{t+1}=\omega+\beta h_{t}+\gamma \varepsilon_{t}^{2}+\delta S_{t}^{-} \varepsilon_{t}^{2},
$$

where $S_{t}^{-}=1$ if $\varepsilon_{t}<0$, and $S_{t}^{-}=0$ if $\varepsilon_{t} \geq 0$, and $\beta+\gamma+\frac{1}{2} \delta<1, \omega>0, \beta \geq 0, \gamma \geq 0$, and $\gamma+\delta \geq 0$.

- the APARCH(1,1) model, (Ding, Granger, and Engle, 1993)

$$
h_{t+1}^{\frac{\delta}{2}}=\omega+a_{1}\left(\left|\varepsilon_{t}\right|-\gamma_{1} \varepsilon_{t}\right)^{\delta}+b_{1} h_{t}^{\frac{\delta}{2}}
$$

where $\delta>0,\left|\gamma_{1}\right|<1$.

The selected GARCH models are the most widely used in the literature, as, they incorporate both the time varying nature of the volatility and leverage effect. The Lévy and jumps processes introduced in the different equations are consistent with the existence of extreme events and their impact on the slowly decreasing tails of financial asset returns.

\subsection{The Stochastic Discount Factors and the corresponding risk neu- tral dynamics}

A discrete time approach to option pricing implies the non-uniqueness of the pricing measure. This uniqueness can be obtained by specifying a stochastic discount factor. However, there has until now been no criterion to decide on a specific pricing kernel. In order to highlight the specific ability of Lévy processes to deliver a realistic pricing for options, we use two existing pricing kernels: first, the exponential affine stochastic discount factor, also known as the Esscher transform by Gerber and Shiu (1994b); second, the extended Girsanov principle developed by Elliott and Madan (1998) based on the existence of a Minimal Entropy Martingale Measure, as in Fujiwara and Miyahara (2003). By doing so, we will able to interpret the interest of Lévy processes when it comes to option pricing, beyond the choice of a pricing kernel.

\subsubsection{Pricing options with exponential affine stochastic discount factors}

In this Section we consider that the stochastic discount factor $M_{t}$ is characterized by the Esscher transform $\forall t \in\{0, \ldots, T-1\}$ :

$$
M_{t, t+1}=\exp \left(\theta_{t+1} Y_{t+1}+\xi_{t+1}\right)
$$

where $Y_{t+1}$ is introduced in (1) or in (2), and where $\theta_{t+1}$ and $\xi_{t+1}$ are $\mathcal{F}_{t}$-measurable random variables. We need to compute explicitly $\left(\theta_{t+1}, \xi_{t+1}\right)$. By writing the pricing formula for the risk-free and risky asset at different dates, we obtain two arbitrage free conditions that induce restrictions on the relationship between the SDF and the historical distribution. More precisely, the constraints are:

$$
\left\{\begin{array} { l } 
{ E ^ { \mathbb { P } } [ M _ { t , t + 1 } e ^ { r } | \mathcal { F } _ { t } ] = 1 } \\
{ E ^ { \mathbb { P } } [ M _ { t , t + 1 } \operatorname { e x p } ( Y _ { t + 1 } ) | \mathcal { F } _ { t } ] = 1 }
\end{array} \Longleftrightarrow \left\{\begin{array}{l}
\varphi_{t}^{\mathbb{P}}\left(\theta_{t+1}+1\right)=e^{r} \varphi_{t}^{\mathbb{P}}\left(\theta_{t+1}\right) \\
\varphi_{t}^{\mathbb{P}}\left(\theta_{t+1}+1\right)=\exp \left(-\xi_{t+1}\right)
\end{array}\right.\right.
$$


where $\varphi_{t}^{\mathbb{P}}(u)=E^{\mathbb{P}}\left[e^{u Y_{t+1}} \mid \mathcal{F}_{t}\right]$ is the moment generating function under the historical distribution $\mathbb{P}$ of $Y_{t+1}$, given $\mathcal{F}_{t}$. This system admits a unique solution $\left(\theta_{t+1}, \xi_{t+1}\right)$. Thus, to characterize the process $\left(Y_{t}\right)_{t}$ under the risk neutral distribution that is denoted $\mathbb{Q}$, the following equation must be solved:

$$
E^{\mathbb{P}}\left[M_{t, t+1} \Phi_{t+1} \mid \mathcal{F}_{t}\right]=e^{-r} E^{\mathbb{Q}}\left[\Phi_{t+1} \mid \mathcal{F}_{t}\right]
$$

where $\Phi_{t+1}$ is the payoff at $t+1$.

Then the distribution of $Y_{t}$ under the risk neutral distribution $\mathbb{Q}$ is obtained through the moment generating function under $\mathbb{Q}$ of $Y_{t+1}$, given $\mathcal{F}_{t}$.

Proposition 2.1 The associated unique risk-neutral conditional distribution $\mathbb{Q}$ of $Y_{t+1}$, given $\mathcal{F}_{t}$, has a probability density function (p.d.f.) with respect to the corresponding historical distribution given by $\frac{M_{t, t+1}}{E_{\mathbb{P}}\left[M_{t, t+1} \mid \mathcal{F}_{t}\right]}$ and a moment generating function given by:

$$
\varphi_{t}^{\mathbb{Q}}(v)=\frac{\varphi_{t}^{\mathbb{P}}\left(\theta_{t+1}+v\right)}{\varphi_{t}^{\mathbb{P}}\left(\theta_{t+1}\right)} .
$$

This proposition derives from Proposition 2 in Chorro et al. (2012) as we introduce the stochastic process:

$$
\left(L_{t}=\prod_{k=1}^{t} \frac{e^{\theta_{k} Y_{k}}}{\varphi_{k-1}^{\mathbb{P}}\left(\theta_{k}\right)}\right)_{t \in\{1, \ldots, T\}}
$$

that is a martingale under $\mathbb{P}$.

Generally speaking, we obtain $\theta_{t}$ and $\xi_{t}$ solving (9). We derived the unicity of $\theta_{t}$ in the annex and we see its existence in Gerber and Shiu (1994a, page 664).

Note that the equation (12) corresponds to the risk neutral measure obtained through the conditional Esscher transformed measure with respect to the filtration $\mathcal{F}_{t}$. The relationship (11) is fundamental as it gives explicitely the conditional distribution of the $\log$ returns under the risk neutral distribution $\mathbb{Q}$. We now adapt Proposition 2.1 to the three types of Lévy process-based models presented earlier.

- In the particular case of model (1) when the innovations $z_{t}$ are characterized by a $N I G(\mu, \alpha, \beta, \delta)$ distribution with triplet $(c, 0, U),{ }^{2}$ then $\left(\sqrt{h_{t}} z_{t}\right)_{t}$ is a form of NIG process with triplet $\left(c^{\prime}, 0, \nu\right)$, with:

$$
c^{\prime}=c \sqrt{h_{t}}+\int_{\mathbb{R}} z \sqrt{h_{t}}\left(1_{\left\{|z| \leq \frac{1}{\sqrt{h_{t}}}\right\}}(z)-1_{\{|z| \leq 1\}}(z)\right) u(z) d z,
$$

then we will use the following result:

Proposition 2.2 Given $\mathcal{F}_{t-1}$, assuming that the process $\left(Y_{t}\right)_{t}$ is defined in (1), un$\operatorname{der} \mathbb{Q}$, the process $\left(\sqrt{h_{t}} z_{t}\right)_{t}$ is again a Lévy process with triplet $\left(c^{\mathbb{Q}}, 0, \nu^{\mathbb{Q}}\right)$ where:

$$
c^{\mathbb{Q}}=c \sqrt{h_{t}}-\int_{\{|z| \leq 1\}} z \sqrt{h_{t}} u(z) d z+\int_{\left\{|z| \leq \frac{1}{\sqrt{h_{t}}}\right\}} z \sqrt{h_{t}} e^{\theta_{t} \sqrt{h_{t}} z} u(z) d z
$$

\footnotetext{
${ }^{2}$ The full characterization of the risk neutral dynamics is provided in the appendices.
} 
and

$$
\nu^{\mathbb{Q}}(d x)=e^{\theta_{t} x} u\left(\frac{x}{\sqrt{h_{t}}}\right) \frac{d x}{\sqrt{h_{t}}} .
$$

The unique value of $\theta_{t}$ is the solution of:

$$
\tilde{m}_{t}+\mu \sqrt{h_{t}}+\delta\left(\sqrt{\alpha^{2}-\left(\beta+\theta_{t} \sqrt{h_{t}}\right)^{2}}-\sqrt{\alpha^{2}-\left(\beta+\left(\theta_{t}+1\right) \sqrt{h_{t}}\right)^{2}}\right)=r,
$$

and the distribution of $Y_{t}$ given $\mathcal{F}_{t-1}$ under $\mathbb{Q}$ is a (Chorro et al. (2012))

$$
N I G\left(\widetilde{m}_{t}+\mu \sqrt{h_{t}}, \frac{\alpha}{\sqrt{h_{t}}}, \frac{\beta}{\sqrt{h_{t}}}+\theta_{t}, \delta \sqrt{h_{t}}\right) .
$$

We can check that under $\mathbb{Q}$, the conditional mean return is not $\widetilde{m}_{t}$ but $\widetilde{m}_{t}+\widetilde{m}_{t}^{\text {shift }}$ with $\widetilde{m}_{t}^{\text {shift }}=\int_{\mathbb{R}} z \sqrt{h_{t}}\left(e^{\theta_{t} \sqrt{h_{t}} z}-1\right) U(d z)$. Moreover, the process $\left(Y_{t}\right)_{t}$ is no longer centered and its variance is not $h_{t}$ but $\operatorname{var}\left(Y_{t}\right)=h_{t}\left(\sigma^{2}+\int_{\mathbb{R}} z^{2} e^{\theta_{t} \sqrt{h_{t}} z} U(d z)\right)$.

- When the returns are governed by model (2), the moment generating functions under $\mathbb{P}$ and $\mathbb{Q}$ are given in the following proposition.

Proposition 2.3 Given $\mathcal{F}_{t-1}$, the moment generating functions under $\mathbb{P}$ and $\mathbb{Q}$ of the process $\left(Y_{t}\right)_{t}$ introduced in (2) are respectively:

$$
\begin{gathered}
\varphi_{t-1}^{\mathbb{P}}(u)=\exp \left[u \widetilde{m}_{t}+\frac{1}{2} h_{t} u^{2}+\lambda\left(e^{\mu u+\frac{1}{2} \sigma^{2} u^{2}}-1\right)\right] \text { and } \\
\varphi_{t-1}^{\mathbb{Q}}(u)=\exp \left[u \widetilde{m}_{t}+h_{t}\left(\theta_{t} u+\frac{1}{2} u^{2}\right)+\lambda e^{\mu \theta_{t}+\frac{1}{2} \sigma^{2} \theta_{t}^{2}}\left(e^{\mu u+\sigma^{2}\left(\theta_{t} u+\frac{1}{2} u^{2}\right)}-1\right)\right] .
\end{gathered}
$$

The unique value of $\theta_{t}$ is the solution of:

$$
\widetilde{m}_{t}+\frac{1}{2} h_{t}\left(2 \theta_{t}+1\right)+\lambda\left[e^{\mu\left(\theta_{t}+1\right)+\frac{1}{2} \sigma^{2}\left(\theta_{t}+1\right)^{2}}-e^{\mu \theta_{t}+\frac{1}{2} \sigma^{2} \theta_{t}^{2}}\right]=r .
$$

Therefore, the risk neutral dynamics for the returns $\left(Y_{t}\right)_{t}$ belongs to the same family introduced under the historical measure $\mathbb{P}$, with

$$
z_{t} \stackrel{\mathbb{Q}}{\sim} N\left(\sqrt{h_{t}} \theta_{t}, 1\right), \quad N_{t} \stackrel{\mathbb{Q}}{\sim} \mathcal{P}\left(\lambda e^{\mu \theta_{t}+\frac{1}{2} \sigma^{2} \theta_{t}^{2}}\right) \quad \text { and } \mathrm{V}_{\mathrm{j}} \stackrel{\mathbb{Q}}{\sim} \mathrm{N}\left(\mu+\sigma^{2} \theta_{\mathrm{t}}, \sigma^{2}\right)^{3} .
$$

The characteristic triplets are $\left(\widetilde{m}_{t}+h_{t} \theta_{t}, h_{t}, \nu^{\mathbb{Q}}\right)$, where $\nu^{\mathbb{Q}}(x)=\lambda e^{\theta_{t} x} f(x) d x$ and $f$ is the density of the normal distribution with mean $\mu$ and variance $\sigma^{2}$. Consequently, under $\mathbb{Q}$, the returns distribution is obtained shifting the conditional mean and the conditional variance.

- When the returns are governed by model (3), the moment generating functions under $\mathbb{P}$ and $\mathbb{Q}$ are given in the following proposition.

\footnotetext{
${ }^{3}$ The proof of this proposition is included in the annex
} 
Proposition 2.4 Given $\mathcal{F}_{t-1}$, the moment generating functions under $\mathbb{P}$ and $\mathbb{Q}$ of the process $\left(Y_{t}\right)_{t}$ introduced in (3) are respectively:

$$
\begin{gathered}
\varphi_{t-1}^{\mathbb{P}}(u)=\exp \left[u \widetilde{m}_{t}+\frac{1}{2} h_{t} u^{2}+\lambda\left(e^{\mu \sqrt{h_{t}} u+\frac{1}{2} \sigma^{2} h_{t} u^{2}}-1\right)\right] \text { and } \\
\varphi_{t-1}^{\mathbb{Q}}(u)=\exp \left[u \widetilde{m}_{t}+h_{t}\left(\theta_{t} u+\frac{1}{2} u^{2}\right)+\lambda e^{\mu \sqrt{h_{t}} \theta_{t}+\frac{1}{2} \sigma^{2} h_{t} \theta_{t}^{2}}\left(e^{\mu \sqrt{h_{t}} u+\sigma^{2} h_{t}\left(\theta_{t} u+\frac{1}{2} u^{2}\right)}-1\right)\right] .
\end{gathered}
$$

The unique value of $\theta_{t}$ is the solution of:

$$
\widetilde{m}_{t}+\frac{1}{2} h_{t}\left(2 \theta_{t}+1\right)+\lambda\left[e^{\mu \sqrt{h_{t}}\left(\theta_{t}+1\right)+\frac{1}{2} \sigma^{2} h_{t}\left(\theta_{t}+1\right)^{2}}-e^{\mu \sqrt{h_{t}} \theta_{t}+\frac{1}{2} \sigma^{2} h_{t} \theta_{t}^{2}}\right]=r .
$$

Therefore, the risk neutral dynamics for the returns $\left(Y_{t}\right)_{t}$ belongs to the same family introduced under the historical measure $\mathbb{P}$, with

$$
z_{t} \stackrel{\mathbb{Q}}{\sim} N\left(\sqrt{h_{t}} \theta_{t}, 1\right), \quad N_{t} \stackrel{\mathbb{Q}}{\sim} \mathcal{P}\left(\lambda e^{\mu \sqrt{h_{t}} \theta_{t}+\frac{1}{2} \sigma^{2} h_{t} \theta_{t}^{2}}\right) \text { and } \mathrm{V}_{\mathrm{j}} \stackrel{\mathbb{Q}}{\sim} \mathrm{N}\left(\mu \sqrt{\mathrm{h}_{\mathrm{t}}}+\sigma^{2} \mathrm{~h}_{\mathrm{t}} \theta_{\mathrm{t}}, \sigma^{2} \mathrm{~h}_{\mathrm{t}}\right)
$$

The characteristic triplets are $\left(\widetilde{m}_{t}+h_{t} \theta_{t}, h_{t}, \nu^{\mathbb{Q}}\right)$, where $\nu^{\mathbb{Q}}(x)=\lambda e^{\theta_{t} x} f(x) d x$ and $f$ is the density of the normal distribution with mean $\mu \sqrt{h_{t}}$ and variance $\sigma^{2} h_{t}$. Consequently, under $\mathbb{Q}$, the returns distribution is again obtained shifting the conditional mean and the conditional variance.

\subsubsection{Pricing options with a Minimal Entropy Martingale Measure}

In this subsection, we derive the risk neutral dynamics of the previous models using another pricing kernel, namely the Extended Girsanov Principle introduced by Elliot and Madan (1998). This approach is based on a multiplicative Doob decomposition of the discounted stock price into a predictable finite variation component and a Martingale one.

Under mild conditions Elliot and Madan (1998) built a risk neutral probability under which the conditional distribution of the discounted stock price is equal to the conditional distribution of its martingale component under the historical probability with changes in the parameters. The drift of the process is the only one being affected by this correction. Nevertheless, unlike the stochastic discount factor that leads to change the value of more than one moment of the return distribution. Beyond this statement drawing a theoretical comparison of the two methods is difficult because of the complexity of the MEMM approach. In the empirical section, we provide an empirical comparison of the two methods which permits to provide the reader with an economic intuition of the two methods ${ }^{4}$.

The Esscher transform and the Minimal Entropy Martingale Measure are equivalent when the conditional distribution of the returns is Gaussian. This equivalence has not been proved for the models considered here.

\footnotetext{
${ }^{4}$ Given the complexity of these risk neutralization, we are not able to pinpoint precisely volatility premium. What is more, given that, the parameter correction associated to the risk neutralisation is time varying, we want be able to present "risk neutral estimates" of the parameters.
} 
- When the returns follow the dynamics specified by equation (1) with NIG-Lévy innovations $\left(z_{t}\right)_{t}$ and assuming that: ${ }^{5}$

(i) $\int_{\{z>1\}} e^{z} e^{\beta\left(e^{z}-1\right)} U(d z)<\infty$;

(ii) $\widetilde{m}_{t}+c^{\prime}+\int_{\{|x| \leq 1\}}\left[\left(e^{x}-1\right) e^{\beta\left(e^{x}-1\right)}-x\right] U\left(\frac{d x}{\sqrt{h_{t}}}\right)+\int_{\{|x|>1\}}\left(e^{x}-1\right) e^{\beta\left(e^{x}-1\right)} U\left(\frac{d x}{\sqrt{h_{t}}}\right)=r$

where

$$
c^{\prime}=\left[c+\int_{\left\{|z| \leq \frac{1}{\sqrt{h_{t}}}\right\}} z U(d z)-\int_{\{|z| \leq 1\}} z U(d z)\right] \sqrt{h_{t}}
$$

Then, we obtain the following result:

Proposition 2.5 Assuming that $\left(Y_{t}\right)_{t}$ is given by (1) with NIG-Lévy innovations $\left(z_{t}\right)_{t}$ under $\mathbb{P}$, then the process $\left(\sqrt{h_{t}} z_{t}\right)_{t}$ is a Lévy process under $\mathbb{Q}$ conditionnally to $\mathcal{F}_{t-1}$, with triplet $\left(c^{\mathbb{Q}}, 0, \nu^{\mathbb{Q}}\right)$ :

$$
\begin{gathered}
c^{\mathbb{Q}}=c \sqrt{h_{t}}-\int_{\{|z| \leq 1\}} z \sqrt{h_{t}} u(z) d z+\int_{\left\{|z| \leq \frac{1}{\sqrt{h_{t}}}\right\}} z \sqrt{h_{t}} e^{\beta\left(e^{z \sqrt{h_{t}}}-1\right)} u(z) d z, \\
\nu^{\mathbb{Q}}(d x)=e^{\beta\left(e^{x}-1\right)} u\left(\frac{x}{\sqrt{h_{t}}}\right) \frac{d x}{\sqrt{h_{t}} .}
\end{gathered}
$$

The characteristic functions of the process $\left(Y_{t}\right)_{t}$ given $\mathcal{F}_{t-1}$ under $\mathbb{P}$ and $\mathbb{Q}$ are:

$$
\begin{aligned}
& \log \phi_{t-1}^{\mathbb{P}}(u)= i u \widetilde{m}_{t}+i u c \sqrt{h_{t}}+ \\
& \int_{\mathbb{R}}\left(e^{i u z \sqrt{h_{t}}}-1-i u z \sqrt{h_{t}} 1_{\{|z| \leq 1\}}\right) U(d z) \text { and } \\
& \log \phi_{t-1}^{\mathbb{Q}}(u)=i u \widetilde{m}_{t}+i u c \sqrt{h_{t}}+ \\
& \int_{\mathbb{R}}\left(e^{i u z \sqrt{h_{t}}}-1-i u z \sqrt{h_{t}} e^{-\beta\left(e^{z \sqrt{h_{t}}}-1\right)} 1_{\{|z| \leq 1\}}\right) e^{\beta\left(e^{z \sqrt{h_{t}}}-1\right)} U(d z) .
\end{aligned}
$$

Thus the characteristic function of $\left(Y_{t}\right)_{t}$ under the risk neutral distribution $\mathbb{Q}$ has the same expression as the historical characteristic function with shift $\sigma^{2} h_{t} \beta$ in the coefficient of $i u$ and integrand $\left(e^{i u z \sqrt{h_{t}}}-1\right) e^{\beta\left(e^{z \sqrt{h_{t}}}-1\right)}$ over $\mathbb{R}$.

In order to compute option prices, we simulate paths of the asset price under the historical measure $\mathbb{P}$. Then, we compute option prices as a weighted average of the payoff for each of the corresponding paths, where the weights are given by the Radon-Nikodym derivative that is defined by:

$$
L_{t}=\left.\frac{d \mathbb{Q}}{d \mathbb{P}}\right|_{\mathcal{F}_{t}}=e^{\beta \widehat{X}_{t}-b t}, \quad \text { with }
$$

\footnotetext{
${ }^{5} \mathrm{~A}$ full mathematical developpement is provided in the appendices.
} 


$$
\begin{gathered}
b=\beta c^{\prime}+\int_{\mathbb{R} \backslash\{0\}}\left[e^{\beta\left(e^{x}-1\right)}-1-\beta x 1_{\{|x| \leq 1\}}\right] U\left(\frac{d x}{\sqrt{h_{t}}}\right), \quad \text { and } \\
\widehat{X}_{t}=X_{t}+\frac{1}{2}\left\langle X^{c}\right\rangle_{t}+\sum_{u \in(0, t]}\left(e^{\Delta X_{u}}-1-\Delta X_{u}\right),
\end{gathered}
$$

where $\left(X_{t}^{c}\right)$ is the continuous martingale part of $\left(X_{t}\right)$, and $\Delta X_{u}=X_{u}-X_{u-}$.

The same representation for the conditional mean as the one obtained using the exponential affine Stochastic Discount Factor can be derived as follows: $\widetilde{m}_{t}^{\text {shift }}=$ $\int_{\mathbb{R}} z \sqrt{h_{t}}\left(e^{\beta\left(e^{z \sqrt{h_{t}}}-1\right)}-1\right) U(d z)$ and $\operatorname{var}\left(Y_{t}\right)=h_{t}\left(\sigma^{2}+\int_{\mathbb{R}} z^{2} e^{\beta\left(e^{z \sqrt{h_{t}}}-1\right)} U(d z)\right)$.

- When the returns $\left(Y_{t}\right)_{t}$ are governed by model (2) under $\mathbb{P}$, then the risk neutral dynamics are characterized as follows:

Proposition 2.6 Let the price process $\left(S_{t}\right)_{t}$ be defined by model (2), assuming that the condition (i) above holds, and there exists $\beta \in \mathbb{R}$ verifying:

$$
\begin{aligned}
\widetilde{m}_{t}+\left(\frac{1}{2}+\beta\right) h_{t}+ & \lambda \int_{\{|z| \leq 1\}}\left[\left(e^{z}-1\right) e^{\beta\left(e^{z}-1\right)}-z\right] f(z) d z \\
& +\lambda \int_{\{z>1\}}\left(e^{z}-1\right) e^{\beta\left(e^{z}-1\right)} f(z) d z=r
\end{aligned}
$$

where $f$ is the density of the normal distribution with mean $\mu$ and variance $\sigma^{2}$, then the following holds:

1. There exists an unique constant $b$ such that $L_{t}$ is a $\mathbb{P}$-martingale, where $L_{t}=$ $\left.\frac{d \mathbb{Q}}{d \mathbb{P}}\right|_{\mathcal{F}_{t}}=e^{\beta \hat{X}_{t}-b t}, \hat{X}_{t}$ being given by (17) and b verifies:

$$
b=\frac{\beta}{2}(1+\beta) h_{t}+\beta \widetilde{m}_{t}+\lambda \int_{\mathbb{R} \backslash\{0\}}\left[e^{\beta\left(e^{z}-1\right)}-1-\beta z 1_{\{|z| \leq 1\}}\right] f(z) d z .
$$

2. Under $\mathbb{Q}$ the stochastic process $\left(Y_{t}\right)_{t}$ is still a Lévy process, and the characteristics associated with the truncation function $\tau(z):=z 1_{\{|z| \leq 1\}}$ is given by

$$
\begin{gathered}
\left(\beta h_{t}+\widetilde{m}_{t}+\lambda \int_{\{|z| \leq 1\}} z\left(e^{\beta\left(e^{z}-1\right)}-1\right) f(z) d z, h_{t}, \nu^{\mathbb{Q}}\right) \text { where, } \\
\nu^{\mathbb{Q}}(d z)=\lambda e^{\beta\left(e^{z}-1\right)} f(z) d z .
\end{gathered}
$$

Fujiwara and Miyahara (2003) proved this result in a particular case. The result provided here has been derived directly from theirs. When the characteristic triplets of the returns $\left(Y_{t}\right)_{t}$ under $\mathbb{Q}$ are known, then we can define the prices under the risk neutral $\mathbb{Q}$.

- When the returns follow the dynamics specified by equation (3) under $\mathbb{P}$, then the risk neutral dynamics are characterized as follows: 
Proposition 2.7 Let the price process $\left(S_{t}\right)_{t}$ be defined by model (3) and there exists $\beta \in \mathbb{R}$ verifying:

$$
\begin{aligned}
\widetilde{m}_{t}+\left(\frac{1}{2}+\beta\right) h_{t}+ & \lambda \int_{\{|z| \leq 1\}}\left[\left(e^{z}-1\right) e^{\beta\left(e^{z}-1\right)}-z\right] f(z) d z \\
& +\lambda \int_{\{z>1\}}\left(e^{z}-1\right) e^{\beta\left(e^{z}-1\right)} f(z) d z=r
\end{aligned}
$$

where $f$ is the density of the normal distribution with mean $\mu \sqrt{h_{t}}$ and variance $\sigma^{2} h_{t}$, then:

There exists a unique constant $b$ such that $L_{t}$ is a $\mathbb{P}$-martingale, where $L_{t}=\left.\frac{d \mathbb{Q}}{d \mathbb{P}}\right|_{\mathcal{F}_{t}}=$ $e^{\beta \hat{X}_{t}-b t}, \hat{X}_{t}$ being given by (17) and b verifying:

$$
b=\frac{\beta}{2}(1+\beta) h_{t}+\beta \widetilde{m}_{t}+\lambda \int_{\mathbb{R} \backslash\{0\}}\left[e^{\beta\left(e^{z}-1\right)}-1-\beta z 1_{\{|z| \leq 1\}}\right] f(z) d z .
$$

\section{Empirical Results}

This Section is devoted to an empirical assessment of the option pricing models presented above.

\subsection{The dataset}

We use two types of data. First, we use daily returns on the S\&P 500 and on the CAC 40 indices. These returns are computed from closing prices. Both datasets start on March 1, 1990 and end on October 26, 2007. Descriptive statistics are available in Table 1.

[Table 1 here]

In spite of very similar maximum and minimum values for both series of returns, the first four moments are nonetheless different: the S\&P 500 returns have a more negative skewness and a higher kurtosis than CAC 40 returns, suggesting a more important tail activity in the US market. Beyond these differences, both empirical distributions have fat tails, driving us to investigate what kind of conditional distribution could provide us with an interesting statistical fit.

Table 2 presents the results of two adequation tests: the Kolmogorov and Smirnov test and the Anderson and Darling test. We apply these tests to the residuals of the three different GARCH models used in this article: the EGARCH, the GARCH-GJR and the APARCH models. Our test results indicate a conditionally Gaussian model would not make it possible to replicate the residuals'tail behavior, as shown on Figure (1) and (2). In contrast, every conditional disrtibution used for our models are found to be consistent with our datasets. This is an essential starting point for our option pricing approach.

[Figure 1 here] 
[Figure 2 here]

[Table 2 here]

A second dataset is made of option prices. This dataset starts on December 6, 2006 and ends on October 26, 2007. During this period, the CAC 40 and the S\&P 500 were sufficiently liquid to provide us with reliable prices. It contains call option prices for a large range of moneyness and maturities. These options are marketed options for the next four quarterly maturities: March, June, September and December. These contracts are the most liquid ones over an extended period of time such as ours.

[Table 3 here]

Table 3 presents a breakdown of the number of option contracts used depending on their moneyness. The moneyness is defined as the ratio between the forward price of the underlying asset and the option's strike price. The number of option prices is higher for moneyness between 0.9 and 1 , which implies a higher accuracy of our results for this type of options. The lowest number of option prices is obtained for moneynesses higher than 1.1. The results presented later for this type of moneyness should therefore be treated with caution. The total number of option prices used in our empirical investigations is 33,400 in the S\&P 500 and 52,292 in the CAC 40 case. This amounts to an average of 163 prices per day in the CAC 40 case and of 104 in the S\&P 500 case

\subsection{Estimation}

To estimate the parameters of models (1)-(7), we first select a subsample containing 4000 working days and ending on a date $t$. Using a Quasi Maximum Likelihood estimation strategy $^{6}$ (Gourieroux et al. 1984a), we estimate the parameters driving the volatility dynamics $h_{t+1}$. We then move to date $t+1$, in a rolling window fashion, and reestimate the parameters from the last 4000 observations. Tables 4 to 6 provide the values of the average estimated parameters of the EGARCH, GARCH-GJR and APARCH models respectively with their standard deviations.

[Table 4 here]

[Table 5 here]

[Table 6 here]

\footnotetext{
${ }^{6}$ Our approach is very different from the methodology in Christoffersen et al. $(2006,2011)$, and Duan et al. (2007): our primary interest lies in the comparison of different existing kernels (exponential affine and MEMM) jointly with three different empirically grounded models. One key difference with the three cited papers is that we do not use options in the estimation procedure. With this choice, we intend to shed light on the ability of these pricing kernels and models to replicate the empirical behavior of options, given that we compare the computed prices to the actual ones from our option dataset.
} 
Our estimates are statistically significant at a 5\% risk level. The comparison of the results in the two cases deliver interesting insights regarding the behavior of each market: consistently with the stronger S\&P 500 skewness found in the descriptive statistics, we obtain stronger leverage in our EGARCH, GARCH-GJR and APARCH estimates for the S\&P 500. For example, in the GARCH-GJR model, $\delta$ controls the leverage effect: in the S\&P case its estimation is equal to 0.104 whereas in the CAC case it is equal to 0.072 . Beyond this difference, most of our estimates are pretty similar.

Then, using the previous volatility dynamics, we estimate the unknown parameters $(\alpha, \beta, \delta, \mu)$ of the NIG distribution for model (1), and the parameters $\lambda,\left(\mu, \sigma^{2}\right)$ of the Poisson and the normal distributions for models (2) and (3), by maximum likelihood estimation. Tables 7, 8 and 9 provide the average estimated parameters with their standard deviation for the NIG distribution and the Poisson based distributions, using the EGARCH, GARCH-GJR and APARCH's residuals obtained in the previous steps.

\section{[Table 7 here]}

[Table 8 here]

[Table 9 here]

Here again, the estimated parameters are different from zero. We obtain minor differences between the parameters estimated in the S\&P and in the CAC cases. Over all the estimated conditional distributions, the CAC 40 residuals seem to be more agitated than the S\&P ones. For example in the NIG case, $\alpha$ controls the tails of the distribution: the higher the $\alpha$ and the fatter the tails. In the CAC case, $\alpha$ is estimated to be equal to 2.5 roughly speaking whereas in the $\mathrm{S} \& \mathrm{P}$ case it is closer to 1.8. A similar message is obtained from Table 8: the variance of the jumps in the CAC case is broadly speaking three times as big as that of the S\&P 500 .

\subsection{Pricing results}

Now, using the previous estimates for the time series models presented earlier, we compute option prices using two ways to obtain the risk neutral distribution: the MEMM and the affine pricing kernel methodologies. We remind the reader that we use a rolling window analysis, estimating first the parameters using 4000 days of returns and then turning the historical distribution into a risk neutral one, from which we compute option prices. We then compare these prices to the actual market prices. By doing so, we hope that when the selected data generating process is as close as possible to the reality of financial markets, the option prices derived from the "risk neutralization" are not too far away from market prices themselves.

Let $\bar{P}(t, T, K)$ be the price of a call option on date $t$ with a maturity equal to $T$ and a strike price equal to $K$. Insofar as we cannot compute the moment generating function of sums of returns under the various models considered here, we compute option prices 
by Monte Carlo simulation. These simulations are done using the Duan and Simonato (1998)'s method to impose martingality within the sampled processes.

We now present the two ways to obtain these prices with respect to the pricing kernels that we use:

- The price of the European call option under $\mathbb{Q}$ obtained using an exponential affine Stochastic Discount Factor is:

$$
\bar{P}(t, T, K)=\frac{1}{N} \sum_{i=1}^{N} e^{-r(T-t)} f\left(Y_{T}^{i}\right)
$$

where $f\left(Y_{T}^{i}\right)=\left(S_{T}^{i}-K\right)^{+}$is the payoff with $S_{T}^{i}=S_{t} e^{\sum_{k=t+1}^{T} Y_{k}^{i}}, T$ is the maturity, $K$ is the strike price, and $\left(Y_{T}^{i}\right)$ for $i=1, \ldots, N$ are independent realizations of $Y_{T}$ under $\mathbb{Q}$.

- The price of the European call option under $\mathbb{Q}$ obtained using the Minimal Entropy Martingale Measure is given by:

$$
\bar{P}(t, T, K)=\frac{1}{N} \sum_{i=1}^{N} e^{-r(T-t)} L_{T}^{i} f\left(Y_{T}^{i}\right)
$$

where $\left(Y_{T}^{i}\right)$ for $i=1, \ldots, N$ are independent realizations of $Y_{T}$ under $\mathbb{P}$ and $L_{T}^{i}$ are realizations of $L_{T}$ introduced in (16).

For each set of option prices we run $N=10,000$ Monte Carlo simulations. In order to compare the pricing performances of the EGARCH, GJR and APARCH respectively with NIG and Poisson distributions as defined in (1)-(7) using both the Esscher transform (ESS) and the Minimal Entropy Martingale Measure (MEMM), we use the average absolute relative pricing errors criterion given by:

$$
A A R P E=\frac{1}{M} \sum_{t=\tau_{1}}^{\tau_{2}} \sum_{j=1}^{J_{t}} \sum_{i=1}^{G_{t, j}}\left|\frac{P\left(t, T_{j}, K_{i}\right)-\bar{P}\left(t, T_{j}, K_{i}\right)}{P\left(t, T_{j}, K_{i}\right)}\right|,
$$

where $P\left(t, T_{j}, K_{i}\right)$ is the corresponding quoted market option price, $M$ is the total number of option prices involved in the computation of the criterion, $J_{t}$ is the number of call option maturities $T_{j}$ available at time $t$ ( $t$ runs from December 6, $2006\left(\tau_{1}\right)$ to October 26, $2007\left(\tau_{2}\right)$ ), and $G_{t, j}$ is the number of strikes $K_{i}$ available at time $t$ for this particular maturity $T_{j}$. In Tables 10 and 11, we provide the pricing errors for different moneyness for the CAC 40 and the S\&P 500 using the three previous models respectively with the Esscher transform and with the Minimal Entropy Martingal Measure. Results have been gathered by time to maturity buckets: 7 days $\leq \mathrm{T}-\mathrm{t} \leq 30$ days, 3 months $\leq \mathrm{T}-\mathrm{t} \leq 6$ months, and $T-t>6$ months.

[Table 10 here] 


\section{[Table 11 here]}

Tables 10 and 11 deliver the following messages:

(1) First of all, despite the fact that the various models' parameters have not been estimated from option prices, the scale of the errors obtained is rather small for both the S\&P 500 and the CAC 40. In the S\&P 500 case for example, when the time to maturity is between 7 and 30 days and with a moneyness below 1 , the AARPE ranges between $10.5 \%$ and $21.6 \%$. In the CAC 40 case, with a time to maturity greater than 6 months and for a moeyness below 1 again, the AARPE ranges from $5 \%$ to $38.1 \%$ (but for the model (3) whose pricing errors are larger than usual). These results are close to what is obtained with estimation methodologies that use option prices directly, as presented in Christoffersen et al. (2006) or Barone Adesi et al. (2008), and Miyahara and Moriwaki (2009). This is a very positive message for the potential use of these discrete time option pricing approaches: when the data generating process used in the option pricing model aims at being realistic, the option prices obtained can be realistic themselves. This is essential in the case of options for which liquidity is low - which happens even for S\&P 500 options during liquidity squeezes - or for options for which there is no organized market.

(2) Then, comparing results with each others, we obtain very positive results in the case of the model (2)-EGARCH, both for the S\&P 500 and for CAC 40 datasets. It is selected to be the best model - as measured with the AARPE criteria - in the S\&P 500 case for a moneyness below 1 for the three types of time to maturity retained here ${ }^{7}$. For a moneyness higher than 1, the model (2)-EGARCH dominates the other competitors when the time to maturity is either between 7 and 30 days or higher than 6 months. In the case of a time to maturity between 3 and 6 months, the model (3)-EGARCH dominates. However, here again, the model (2)-EGARCH is not far away from that. Finally, and to be fair, the model (3)-EGARCH and the model (2)-EGARCH actually deliver fairly comparable results across our pricing results: the fact that jumps have an impact on the conditional variance or not does not seem to have a strong impact on the option pricing results.

Moving to the CAC 40 results, we find a similar conclusion when it comes to moneyness below 1: either the model (2)-EGARCH or the model (3)-EGARCH deliver usually the best results. This holds but for short term options for which the APARCH based model (3) and model (2) deliver the best results. For moneyness higher than 1 , we obtain in the CAC 40 case something different from what we obtained in the S\&P 500 case: here, it seems that the NIG-based model dominates the others when the time to maturity is higher than 3 months. However, the limited number of observations for this type of options prevents us from reaching a more definitive conclusion.

\footnotetext{
${ }^{7}$ For a time to maturity greater than 6 months and a moneyness between 0.9 and 1, the model (2)APARCH actually dominates, with an AARPE equal to 15.5\%, but the model (2)-EGARCH is really close to this, with an AARPE equal to $15.8 \%$.
} 
(3) A last message conveyed by our empirical results is related to the pricing kernels used here. When comparing the pricing results obtained with each of the two possibilities considered here, we globally find limited differences in terms of pricing accuracy. When focusing for example on the EGARCH model with Gaussian jumps in the CAC 40 case, we obtain an AARPE of $6.6 \%$ for options with a time to maturity higher than 6 months and a moneyness between 0.8 and 0.9 with the affine pricing kernel, when the MEMM yields 7.1\%. On top of that, we find that for some models with specific time to maturities and moneyness that the affine pricing kernel dominates the MEMM, whereas the contrary is also possible in some other cases. This can be explained by two different factors: first, the martingalization method of Duan and Simonato (1998) have a strong impact on the option pricing results. For example, Chorro et al. (2010a) empirically show how this method helps computing accurate option prices without having to assume any pricing kernel. Beyond that, we compared these pricing kernels through the risk aversion function that they imply ${ }^{8}$.

From Proposition 2.1 and relationship (16), the ratio of the risk-neutral conditional distribution $f_{t}^{*}$ of $\left(Y_{t+1}, \ldots, Y_{T}\right)$ given $\mathcal{F}_{t}$ to the corresponding historical conditional distribution $f_{t}$ is proportional to the stochastic discount factor ${ }^{9} M_{t, T}$ (or marginal rate of substitution (MRS)) between consumption at dates $t$ and $T$ of the representative agent:

$$
\zeta_{t}\left(S_{T}\right) \equiv \frac{f_{t}^{*}\left(S_{T}\right)}{f_{t}\left(S_{T}\right)} \propto M_{t, T}=\frac{U^{\prime}\left(S_{T}\right)}{U^{\prime}\left(S_{t}\right)}
$$

So, we can write the Arrow-Pratt (Arrow, 1964; Pratt, 1964) measure of absolute risk aversion $(A R A) \rho_{t}\left(S_{T}\right)$ by:

$$
\rho_{t}\left(S_{T}\right) \equiv-\frac{\zeta_{t}^{\prime}\left(S_{T}\right)}{\zeta_{t}\left(S_{T}\right)}=-\frac{U^{\prime \prime}\left(S_{T}\right)}{U^{\prime}\left(S_{T}\right)}=\frac{f_{t}^{\prime}\left(S_{T}\right)}{f_{t}\left(S_{T}\right)}-\frac{f_{t}^{*^{\prime}}\left(S_{T}\right)}{f_{t}^{*}\left(S_{T}\right)}=-d \log M_{t, T}
$$

In the Black and Scholes model, the marginal rate of substitution and the absolute risk aversion are:

$$
\zeta_{B S, t}\left(S_{T}\right)=C\left(\frac{S_{T}}{S_{t}}\right)^{-a} \quad \text { and } \quad \rho_{\mathrm{BS}, \mathrm{t}}\left(\mathrm{S}_{\mathrm{T}}\right)=\frac{\mathrm{a}}{\mathrm{S}_{\mathrm{T}}}
$$

where $C$ denotes a constant and $a=(\mu-r) / \sigma^{2}$.

We estimate risk aversion from equation (23): using 10,000 Monte Carlo simulations of three months samples of returns under the historical and risk neutral distribution, we estimate $f_{t}^{*}\left(S_{T}\right)$ and $f_{t}\left(S_{T}\right)$ using a non parametric estimator based on a Gaussian kernel. Once these densities have been estimated, we deduce the absolute risk aversion using the relation presented in equation (23). It has been applied in the literature by Jackwerth (2000), Rosenberg and Engle (2002) and more recently in Chevallier et al. (2008) .

\footnotetext{
${ }^{8}$ Given the path dependency of the "risk neutralization" that these pricing kernels imply, it is not possible to derive a closed form expression for the pricing kernel between date $t$ and date $T$. We however estimate the three months ahead pricing kernel in the S\&P 500 and in the CAC 40 cases using the full sample parameter estimates, using then Monte Carlo simulations.

${ }^{9}$ On this relation see Ait Sahalia and Lo (1998, 2000)
} 
In Figure 3, we present the Absolute Risk Aversion using the affine pricing kernel (solid lines), the MEMM pricing kernel (dashed) and the Absolute Risk Aversion of the Black and Scholes model (dotted) of the CAC 40 and the S\&P 500 indexes for 3 months time to maturity. Each panel corresponds to the three models associated to the three types of GARCH models.

[Figure 3 here]

For each type of models and pricing kernels, we obtain a decreasing risk aversion when future wealth (that is the moneyness) is increasing, hence being consistent with the basic intuitions regarding this economic parameter. The analysis of these graphs provides us with two main conclusions: first, for both pricing kernels the slope of the risk aversion is less steep that in the Black and Scholes case. With these pricing kernels, risk aversion vanishes less rapidly when the moneyness increases. Second - and more importantly - both pricing kernels look very similar when they are compared on this common scale: for most of these graphs the solid and the dashed lines overlap. This should partly explain why the choice of the pricing kernel seems to have a limited impact on the pricing errors. In some sense - and given that we use the two main pricing kernels used in the literature - this delivers the message that the specification of the data generating process under the historical distribution may matter more than the specification of the representative agent's risk preferences.

\section{Conclusion}

This article presents different types of discrete time Lévy processes for option pricing purposes. These models are based on three different volatility dynamics and two different kinds of pricing kernels. We derive the characterization of the risk neutral distribution associated with each of these models. Combining jumps to volatility should provide us with a realistic model of financial returns' dynamics, which is one of the major shortcomings of the Black and Scholes (1973) model. We provide an empirical assessment of these models using a dataset of option prices on the S\&P 500 and the CAC 40 indices. We find that these empirically grounded models provide minimum mispricing errors that are broadly consistent with the results obtained in the literature estimating the models' parameters using option prices. This is all the more interesting as the approach developed here can be thus applied to price options that are illiquid - preventing the use of their prices to estimate the parameters of the pricing model - or to price derivatives for which there is no existing market. A key point of this paper is that the choice of the pricing kernel has a limited impact on the pricing errors, suggesting that the main driver of these errors is rather the underlying time series model rather than the specification of the pricing kernels. We have also observed this fact for hedging: the choice of the pricing kernels does not appear determinant. 


\section{Appendix:}

\subsection{Unicity of $\theta_{t+1}$}

Here, we presente the unicity of $\theta_{t}$ in Proposition 2.1 .

$\theta_{t+1}$ is solution of

$$
\varphi_{t}^{\mathbb{P}}\left(\theta_{t+1}+1\right)=e^{r} \varphi_{t}^{\mathbb{P}}\left(\theta_{t+1}\right)
$$

which is equivalent to

$$
\log \left(\varphi_{t}^{\mathbb{P}}\left(\theta_{t+1}+1\right)\right)-\log \left(\varphi_{t}^{\mathbb{P}}\left(\theta_{t+1}\right)\right)=r
$$

Following Gerber and Shiu (1994), we consider the function

$$
g(\theta)=\log \left(\varphi_{t}^{\mathbb{P}}(\theta+1)\right)-\log \left(\varphi_{t}^{\mathbb{P}}(\theta)\right) .
$$

Then,

$$
\begin{aligned}
g^{\prime}(\theta) & =\frac{1}{\varphi_{t}^{\mathbb{P}}(\theta+1)} \frac{d}{d \theta}\left(\varphi_{t}^{\mathbb{P}}(\theta+1)\right)-\frac{1}{\varphi_{t}^{\mathbb{P}}(\theta)} \frac{d}{d \theta}\left(\varphi_{t}^{\mathbb{P}}(\theta)\right) \\
& =E^{\mathbb{Q}}\left(Y_{t+1}, \theta+1 \mid \mathcal{F}_{t}\right)-E^{\mathbb{Q}}\left(Y_{t+1}, \theta \mid \mathcal{F}_{t}\right),
\end{aligned}
$$

since

$$
\begin{aligned}
\frac{1}{\varphi_{t}^{\mathbb{P}}(\theta)} \frac{d}{d \theta}\left(\varphi_{t}^{\mathbb{P}}(\theta)\right) & =E^{\mathbb{P}}\left[\frac{Y_{t+1} e^{\theta Y_{t+1}}}{\varphi_{t}^{\mathbb{P}}(\theta)} \mid \mathcal{F}_{t}\right] \\
& =E^{\mathbb{Q}}\left(Y_{t+1}, \theta \mid \mathcal{F}_{t}\right),
\end{aligned}
$$

And $E^{\mathbb{Q}}\left(Y_{t+1}, \theta \mid \mathcal{F}_{t}\right)$ is an increasing function of $\theta$. In fact,

$$
\begin{aligned}
\frac{d}{d \theta} E^{\mathbb{Q}}\left(Y_{t+1}, \theta \mid \mathcal{F}_{t}\right) & =\frac{1}{\left[\varphi_{t}^{\mathbb{P}}(\theta)\right]^{2}}\left(E^{\mathbb{P}}\left(Y_{t+1}^{2} e^{\theta Y_{t+1}} \mid \mathcal{F}_{t}\right) \varphi_{t}^{\mathbb{P}}(\theta)-\left[E^{\mathbb{P}}\left(Y_{t+1} e^{\theta Y_{t+1}} \mid \mathcal{F}_{t}\right)\right]^{2}\right) \\
& =E^{\mathbb{Q}}\left(Y_{t+1}^{2} \mid \mathcal{F}_{t}\right)-\left[E^{\mathbb{Q}}\left(Y_{t+1} \mid \mathcal{F}_{t}\right)\right]^{2} \\
& =\operatorname{Var}^{\mathbb{Q}}\left(Y_{t+1}, \theta \mid \mathcal{F}_{t}\right)>0 .
\end{aligned}
$$

So, $g^{\prime}(\theta)>0$, showing that $g(\theta)$ is an increasing. This proves the uniqueness of the solution of equation (25).

\subsection{The general results for the returns modelled by (1)}

(a) - Model with an exponential affine SDF:

Using the relationship (11), we provide for model (1) the moment generating function of the log-returns under $\mathbb{P}$ and $\mathbb{Q}$. 
Proposition 5.1 Given $\mathcal{F}_{t-1}$, the moment generating functions under $\mathbb{P}$ and $\mathbb{Q}$ of the process $\left(Y_{t}\right)_{t}$ introduced in (1) are respectively:

$$
\begin{aligned}
\log \varphi_{t-1}^{\mathbb{P}}(u)= & u \widetilde{m}_{t}+u c \sqrt{h_{t}}+\frac{u^{2}}{2} \sigma^{2} h_{t}+ \\
& \int_{\mathbb{R}}\left(e^{u z \sqrt{h_{t}}}-1-u z \sqrt{h_{t}} 1_{\{|z| \leq 1\}}\right) U(d z)
\end{aligned}
$$

and

$$
\begin{aligned}
\log \varphi_{t-1}^{\mathbb{Q}}(u)= & u \tilde{m}_{t}+u c \sqrt{h_{t}}+\frac{u^{2}}{2} \sigma^{2} h_{t} \theta_{t}+ \\
& \int_{\mathbb{R}}\left(e^{u z \sqrt{h_{t}}}-1-u z \sqrt{h_{t}} e^{-\theta_{t} z \sqrt{h_{t}}} 1_{\{|z| \leq 1\}}\right) e^{\theta_{t} z \sqrt{h_{t}}} U(d z),
\end{aligned}
$$

where $\widetilde{m}_{t}, c, \theta_{t}$ have been defined previously.

The expression of the process under the risk neutral measure is now available:

Proposition 5.2 If the returns $\left(Y_{t}\right)_{t}$ are modelled by (1) under $\mathbb{P}$, then under the risk neutral measure $\mathbb{Q}$, the process $\left(Y_{t}\right)_{t}$ follows the same process where $\left(X_{t}\right)_{t}$ is a Lévy process with triplet $\left(c^{\mathbb{Q}}, \sigma^{2} h_{t}, \nu^{\mathbb{Q}}\right)$ given by:

$$
c^{\mathbb{Q}}=c \sqrt{h_{t}}+\sigma^{2} \theta_{t} h_{t}-\int_{\{|z| \leq 1\}} z \sqrt{h_{t}} U(d z)+\int_{\left\{|z| \leq \frac{1}{\sqrt{h_{t}}}\right\}} z \sqrt{h_{t}} e^{\theta_{t} \sqrt{h_{t}} z} U(d z)
$$

and

$$
\nu^{\mathbb{Q}}(d x)=e^{\theta_{t} x} U\left(\frac{d x}{\sqrt{h_{t}}}\right)
$$

We observe that the conditional distribution of $\left(Y_{t}\right)_{t}$ under $\mathbb{Q}$ belongs to the same family of distributions than under $\mathbb{P}$. Nevertheless, there are changes in the mean and in the variance.

(b) - Model with Minimal Entropy Martingale Measure:

To apply this methodology we recall briefly the framework in which we work, and specify some notations.

Let $G=\left(G_{t}\right)_{t \in[0, T]}, T>0$, be a geometric Lévy process defined on a probability space $(\Omega, \mathcal{F}, \mathbb{P})$, such that:

$$
G_{t}=G_{0} e^{z_{t}}
$$

where $G_{0}>0$ is a constant and $z=\left(z_{t}\right)_{t \in[0, T]}$ is a one-dimensional Lévy process. Let $\left(c, \sigma^{2}, U\right)$ be the characteristics of $\left(z_{t}\right)_{t}$ associated with the truncation function $\tau(z):=$ $z 1_{\{|z| \leq 1\}}$. For a constant $r \in \mathbb{R}$, we set $B_{t}:=e^{r t}$, and we also define $\tilde{G}_{t}:=\frac{G_{t}}{B_{t}}=e^{-r t} G_{t}$ : it is still a geometric Lévy process because $\left(z_{t}-r t\right)$ is a Lévy process, and is the discount 
price process of $G_{t}$. Now if $U=0$, that is if $\left(G_{t}\right)_{t}$ does not have any jump, then this setup is nothing but the Black and Scholes model. Let $\left(\tilde{R}_{t}\right)_{t}$ be the return process for $\left(\tilde{G}_{t}\right)_{t}$. We now introduce a condition on the driving Lévy process $\left(z_{t}\right)$ :

(C): There exists a constant $\beta \in \mathbb{R}$ that satisfies the following conditions (i) and (ii):

(i)

$$
\int_{\{z>1\}} e^{z} e^{\beta\left(e^{z}-1\right)} U(d z)<\infty
$$

(ii)

$$
\begin{aligned}
c+\left(\frac{1}{2}+\beta\right) \sigma^{2}+ & \int_{\{|z| \leq 1\}}\left[\left(e^{z}-1\right) e^{\beta\left(e^{z}-1\right)}-z\right] U(d z) \\
& +\int_{\{|z|>1\}}\left(e^{z}-1\right) e^{\beta\left(e^{z}-1\right)} U(d z)=r .
\end{aligned}
$$

Then, we have the following result due to Fujiwara and Miyahara (2003).

Theorem 5.3 Given the previous return process $\left(\tilde{R}_{t}\right)$, and assuming that the previous condition (C) holds, then

(1) We can define a probability measure $\mathbb{Q}$ on $\mathcal{F}_{T}$ by means of the Esscher transformation:

$$
\left.\frac{d \mathbb{Q}}{d \mathbb{P}}\right|_{\mathcal{F}_{t}}:=\frac{e^{\beta \tilde{R}_{t}}}{E^{P}\left[e^{\beta \tilde{R}_{t}}\right]}:=\frac{e^{\beta \widehat{z}_{t}}}{E_{P}\left[e^{\beta \widehat{z}_{t}}\right]}=e^{\beta \widehat{z}_{t}-b t},
$$

for every $t \in[0, T]$, where $\beta$ is the constant in $(\boldsymbol{C})$, and

$$
\begin{aligned}
\widehat{z}_{t}=z_{t}+\frac{1}{2} \sigma^{2} t & +\int_{(0, t]} \int_{\mathbb{R} \backslash\{0\}}\left(e^{z}-1-z\right) N_{p}(d u d z) \\
=\sigma W_{t}+c_{1} t & +\int_{(0, t]} \int_{\{|z| \leq 1\}}\left(e^{z}-1\right) \tilde{N}_{p}(d u d z) \\
& +\int_{(0, t]} \int_{\{|z|>1\}}\left(e^{z}-1\right) N_{p}(d u d z)
\end{aligned}
$$

with

$$
c_{1}:=\frac{1}{2} \sigma^{2}+c+\int_{\{|z| \leq 1\}}\left(e^{z}-1-z\right) U(d z)
$$

and

$$
b=\frac{\beta}{2}(1+\beta) \sigma^{2}+\beta c+\int_{\mathbb{R} \backslash\{0\}}\left[e^{\beta\left(e^{z}-1\right)}-1-\beta z 1_{\{|z| \leq 1\}}\right] U(d z) .
$$

(2) The stochastic process $\left(z_{t}\right)$ is still a Lévy process under the probability measure $\mathbb{Q}$ defined in (29), and the characteristics associated with the truncation function $\tau(z):=$ $z 1_{\{|z| \leq 1\}}$ are:

$$
\left(\beta \sigma^{2}+c+\int_{\{|z| \leq 1\}} z\left(e^{\beta\left(e^{z}-1\right)}-1\right) U(d z), \sigma^{2}, U^{\mathbb{Q}}\right)
$$

where:

$$
U^{\mathbb{Q}}(d z):=e^{\beta\left(e^{z}-1\right)} U(d z)
$$


We now apply these theoretical results to model (1) we introduced previously for the stock price $\left(S_{t}\right)_{t}$ defined by:

$$
S_{t}=S_{t-1} e^{\tilde{m}_{t}} \exp \left(X_{t}\right)
$$

where $S_{t-1} e^{\widetilde{m}_{t}}$ is $\mathcal{F}_{t-1}$-measurable, and $X_{t}=\sqrt{h_{t}} z_{t}$. Following Fujiwara and Miyahara (2003), we define a new probability measure $\mathbb{Q}$ equivalent to $\mathbb{P}$, introducing the following technical condition on the driving Lévy process $\left(z_{t}\right)$ :

$\left(C_{1}\right)$ : Constants $c$ and $\beta \in \mathbb{R}$ exist and satisfy the following conditions (i) and (ii):

(i)

$$
\int_{\{z>1\}} e^{z} e^{\beta\left(e^{z}-1\right)} U(d z)<\infty
$$

(ii)

$$
\begin{aligned}
\widetilde{m}_{t}+c^{\prime}+\left(\frac{1}{2}+\beta\right) \sigma^{2} h_{t}+ & \int_{\{|x| \leq 1\}}\left[\left(e^{x}-1\right) e^{\beta\left(e^{x}-1\right)}-x\right] U\left(\frac{d x}{\sqrt{h_{t}}}\right) \\
& +\int_{\{|x|>1\}}\left(e^{x}-1\right) e^{\beta\left(e^{x}-1\right)} U\left(\frac{d x}{\sqrt{h_{t}}}\right)=r
\end{aligned}
$$

where:

$$
c^{\prime}=\left[c+\int_{\left\{|z| \leq \frac{1}{\sqrt{h_{t}}}\right\}} z U(d z)-\int_{\{|z| \leq 1\}} z U(d z)\right] \sqrt{h_{t}}
$$

Proposition 5.4 Let the price process $\left(S_{t}\right)_{t}$ be defined by (36), and the condition $\left(C_{1}\right)$ holds, then:

(i) there exists a probability measure $\mathbb{Q}$ defined on $\mathcal{F}_{T}$ :

$$
\begin{gathered}
L_{t}=\left.\frac{d \mathbb{Q}}{d \mathbb{P}}\right|_{\mathcal{F}_{t}}=e^{\beta \widehat{X}_{t}-b t}, \text { with } \\
b=\frac{\beta}{2}(1+\beta) \sigma^{2} h_{t}+\beta c^{\prime}+\int_{\mathbb{R} \backslash\{0\}}\left[e^{\beta\left(e^{x}-1\right)}-1-\beta x 1_{\{|x| \leq 1\}}\right] \nu(d x), \\
\widehat{X}_{t}=X_{t}+\frac{1}{2}\left\langle X^{c}\right\rangle_{t}+\sum_{u \in(0, t]}\left(e^{\Delta X_{u}}-1-\Delta X_{u}\right),
\end{gathered}
$$

where $\left(X_{t}^{c}\right)$ is the continuous martingale part of $\left(X_{t}\right)$, and $\Delta X_{u}=X_{u}-X_{u-}$.

(ii) Under $\mathbb{Q}$, the price process $\left(S_{t}\right)_{t}$ is an exponential-Lévy process $S_{t}=S_{t-1} \exp \left(\widetilde{m}_{t}+X_{t}\right)$ where $\widetilde{m}_{t}$ is introduced in (1), and $\left(X_{t}\right)_{t}$ is a Lévy process with Lévy triplet $\left(c^{\mathbb{Q}}, \sigma^{2} h_{t}, \nu^{\mathbb{Q}}\right)$ given by:

$$
\begin{gathered}
c^{\mathbb{Q}}=c \sqrt{h_{t}}+\sigma^{2} \beta h_{t}-\int_{\{|z| \leq 1\}} z \sqrt{h_{t}} U(d z)+\int_{\left\{|z| \leq \frac{1}{\sqrt{h_{t}}}\right\}} z \sqrt{h_{t}} e^{\beta\left(e^{z \sqrt{h_{t}}}-1\right)} U(d z), \\
\nu^{\mathbb{Q}}(d x)=e^{\beta\left(e^{x}-1\right)} U\left(\frac{d x}{\sqrt{h_{t}}}\right) .
\end{gathered}
$$


Thus the dynamics of $\left(S_{t}\right)_{t}$ under $\mathbb{Q}$ follows the same class of models than under $\mathbb{P}$, with changes inside the parameters of the Lévy process.

From the Theorem 5.3, we can derive the expression of the conditionnal moment generating functions for the process $\left(S_{t}\right)_{t}$ under $\mathbb{P}$ and under $\mathbb{Q}$, and we will use them through Monte Carlo simulations to get option prices.

$$
\begin{aligned}
\log \varphi_{t-1}^{\mathbb{P}}(u)= & u \widetilde{m}_{t}+u c \sqrt{h_{t}}+\frac{u^{2}}{2} \sigma^{2} h_{t}+ \\
& \int_{\mathbb{R}}\left(e^{u z \sqrt{h_{t}}}-1-u z \sqrt{h_{t}} 1_{\{|z| \leq 1\}}\right) U(d z)
\end{aligned}
$$

and

$$
\begin{aligned}
\log \varphi_{t-1}^{\mathbb{Q}}(u)= & u \widetilde{m}_{t}+u c \sqrt{h_{t}}+\frac{u^{2}}{2} \sigma^{2} h_{t} \beta+ \\
& \int_{\mathbb{R}}\left(e^{u z \sqrt{h_{t}}}-1-u z \sqrt{h_{t}} e^{-\beta\left(e^{z \sqrt{h_{t}}}-1\right)} 1_{\{|z| \leq 1\}}\right) e^{\beta\left(e^{z \sqrt{h_{t}}}-1\right)} U(d z)
\end{aligned}
$$

\subsection{The Relationship (10)}

In this subsection, we prove the relationship (10), and the p.d.f of the conditional risk neutral distribution with respect to the corresponding historical distribution in Proposition 2.1.

The price $C_{t}$ at time $t$ of a European derivative asset with payoff $g\left(Y_{t+1}, \ldots, Y_{T}\right)$ at $T$ is

$$
\begin{aligned}
C_{t}(g, T) & =E_{\mathbb{P}}\left[M_{t, t+1} \times \ldots \times M_{T-1, T} \times g\left(Y_{t+1}, \ldots, Y_{T}\right) \mid \mathcal{F}_{t}\right] \\
& =E_{\mathbb{P}}\left[M_{t, T} \times g\left(Y_{t+1}, \ldots, Y_{T}\right) \mid \mathcal{F}_{t}\right]
\end{aligned}
$$

which can be written as

$$
\begin{aligned}
C_{t}(g, T) & =E_{\mathbb{P}}\left[M_{t, T} \mid \mathcal{F}_{t}\right] \times E_{\mathbb{P}}\left[\frac{M_{t, T}}{E_{\mathbb{P}}\left[M_{t, T} \mid \mathcal{F}_{t}\right]} g\left(Y_{t+1}, \ldots, Y_{T}\right) \mid \mathcal{F}_{t}\right] \\
& =e^{-r(T-t)} \times E_{\mathbb{P}}\left[\frac{M_{t, T}}{E_{\mathbb{P}}\left[M_{t, T} \mid \mathcal{F}_{t}\right]} g\left(Y_{t+1}, \ldots, Y_{T}\right) \mid \mathcal{F}_{t}\right]
\end{aligned}
$$

Defining $\frac{d \mathbb{Q}}{d \mathbb{P}}=\frac{M_{t, T}}{E_{\mathbb{P}}\left[M_{t, T}\right]}, C_{t}(g, T)$ can be rewritten as

$$
\begin{aligned}
C_{t}(g, T) & =e^{-r(T-t)} E_{\mathbb{P}}\left[\frac{d \mathbb{Q}}{d \mathbb{P}} g\left(\left(Y_{t+1}, \ldots, Y_{T}\right) \mid \mathcal{F}_{t}\right]\right. \\
& =e^{-r(T-t)} E_{\mathbb{Q}}\left[g\left(Y_{t+1}, \ldots, Y_{T}\right) \mid \mathcal{F}_{t}\right]
\end{aligned}
$$

Thus, the price $C_{t}$ at time $t$ of an asset with payoff $g\left(Y_{t+1}\right)$ at time $t+1$ is:

$$
C_{t}(g)=e^{-r} E_{\mathbb{Q}}\left[g\left(Y_{t+1}\right) \mid \mathcal{F}_{t}\right]
$$

and

$$
\frac{d \mathbb{Q}}{d \mathbb{P}}=\frac{M_{t, t+1}}{E_{\mathbb{P}}\left[M_{t, t+1}\right]}
$$




\subsection{Proof of Proposition 2.3}

The moment generating function under $\mathbb{P}$ of the process $\left(Y_{t}\right)_{t}$ introduced in (2) can be written as

$$
\begin{aligned}
\varphi_{t-1}^{\mathbb{P}}(u) & =E_{\mathbb{P}}\left[\exp \left(u \widetilde{m}_{t}+u X_{t}+u \sum_{j=1}^{N_{t}} V_{j}\right) \mid \mathcal{F}_{t-1}\right] \\
& =\exp \left[u \tilde{m}_{t}+\frac{1}{2} u^{2} h_{t}+\lambda\left(e^{\mu u+\frac{1}{2} \sigma^{2} u^{2}}-1\right)\right],
\end{aligned}
$$

and the moment generating function under $\mathbb{Q}$ is also derived from

$$
\varphi_{t-1}^{\mathbb{Q}}(u)=\frac{\varphi_{t-1}^{\mathbb{P}}\left(\theta_{t}+u\right)}{\varphi_{t-1}^{\mathbb{P}}\left(\theta_{t}\right)}
$$

\subsection{Proof of Proposition 2.4}

The moment generating function under $\mathbb{P}$ of the process $\left(Y_{t}\right)_{t}$ introduced in (3) can be written as

$$
\begin{aligned}
\varphi_{t-1}^{\mathbb{P}}(u) & =E_{\mathbb{P}}\left[\exp \left(u \widetilde{m}_{t}+u X_{t}+u \sum_{j=1}^{N_{t}} V_{j}\right) \mid \mathcal{F}_{t-1}\right] \\
& =\exp \left[u \widetilde{m}_{t}+\frac{1}{2} u^{2} h_{t}+\lambda\left(e^{\mu \sqrt{h_{t}} u+\frac{1}{2} \sigma^{2} h_{t} u^{2}}-1\right)\right],
\end{aligned}
$$

and the moment generating function under $\mathbb{Q}$ is also derived from

$$
\varphi_{t-1}^{\mathbb{Q}}(u)=\frac{\varphi_{t-1}^{\mathbb{P}}\left(\theta_{t}+u\right)}{\varphi_{t-1}^{\mathbb{P}}\left(\theta_{t}\right)} .
$$

\subsection{Proof of Proposition 5.1}

The moment generating function under $\mathbb{P}$ of the process $\left(Y_{t}\right)_{t}$ introduced in (1) is

$$
\begin{aligned}
\varphi_{t-1}^{\mathbb{P}}(u) & =E_{\mathbb{P}}\left[\exp \left(u Y_{t}\right) \mid \mathcal{F}_{t-1}\right] \\
& =E_{\mathbb{P}}\left[\exp \left(u \widetilde{m}_{t}\right) \exp \left(\sqrt{h_{t}} u z_{t}\right) \mid \mathcal{F}_{t-1}\right] \\
& =\exp \left(u \widetilde{m}_{t}\right) E_{\mathbb{P}}\left[\exp \left(u \sqrt{h_{t}} z_{t}\right) \mid \mathcal{F}_{t-1}\right] \text { since } \widetilde{m}_{t} \text { is } \mathcal{F}_{t-1} \text {-measurable } \\
& =\exp \left(u \widetilde{m}_{t}\right) E_{\mathbb{P}}\left[\exp \left(u \sqrt{h_{t}} z_{1}\right)\right] \text { since } z_{t} \text { is i.i.d. } \\
& =\exp \left(u \widetilde{m}_{t}\right) \exp \left[\kappa\left(u \sqrt{h_{t}}\right)\right] \text { by the Lévy-Kintchine formula }
\end{aligned}
$$

and the moment under $\mathbb{Q}$ is derived from (11). 


\subsection{Proof of Proposition 5.2}

In order to prove Proposition 5.2, we first introduce a lemma:

Lemma 5.5 If $z_{t}$ is a Lévy process with triplet $\left(c, \sigma^{2}, U\right)$, then $X_{t}=\sqrt{h_{t}} z_{t}$ is also a Lévy process with triplet $\left(c^{\prime}, \sigma^{2} h_{t}, \nu\right)$ where:

$$
c^{\prime}=c \sqrt{h_{t}}+\int_{\mathbb{R}} x\left(1_{\{|x| \leq 1\}}(x)-1_{S}(x)\right) \nu(d x)
$$

where $S$ is the image by $\sqrt{h_{t}}$ of a unit ball in $\mathbb{R}$ :

$$
S=\left\{\sqrt{h_{t}} z:|z| \leq 1\right\}
$$

and

$$
\nu(d x)=U\left(\frac{d x}{\sqrt{h_{t}}}\right)
$$

Proof Using Lévy-Itô decomposition, $X_{t}=\sqrt{h_{t}} z_{t}$ has the representation:

$$
\begin{gathered}
X_{t}=\sigma \sqrt{h_{t}} W_{t}+c \sqrt{h_{t}} t+\int_{(0, t]} \int_{\{|z| \leq 1\}} z \sqrt{h_{t}} \tilde{N}_{p}(d u d z) \\
+\int_{(0, t]} \int_{\{|z|>1\}} z \sqrt{h_{t}} N_{p}(d u d z) .
\end{gathered}
$$

In order to know the characteristics of $\left(X_{t}\right)_{t}$, we transform the point process $\left(p_{t}\right)_{t}$ defined by $p_{t}:=\Delta X_{t}$, where $\Delta X_{t}=X_{t}-X_{t-}$, into another one $\left(q_{t}\right)_{t}$ by

$$
D_{q}:=D_{p} \quad \text { and } \quad q_{t}:=J\left(p_{t}\right),
$$

where $J(z):=z \sqrt{h_{t}}$ for $z \in \mathbb{R}$, then:

$$
\widehat{N}_{q}(d u d x)=d u \nu(d x)
$$


where $\nu(d x):=U \circ J^{-1}(d x)$ and

$$
\begin{aligned}
X_{t}=\sigma \sqrt{h_{t}} W_{t}+c \sqrt{h_{t}} t & +\int_{(0, t]} \int_{\mathbb{R}} x 1_{S}(x) \tilde{N}_{q}(d u d x) \\
& +\int_{(0, t]} \int_{\mathbb{R}} x 1_{S^{c}} N_{q}(d u d x) \\
\text { where } S=\left\{\sqrt{h_{t}} z:|z| \leq 1\right\} \text { and } S^{c}=\left\{\sqrt{h_{t}} z:|z|>1\right\} & \\
= & \sigma \sqrt{h_{t}} W_{t}+c \sqrt{h_{t}} t+\int_{(0, t]} \int_{\{|x| \leq 1\}} x \tilde{N}_{q}(d u d x)+\int_{(0, t]} \int_{\{|x|>1\}} x N_{q}(d u d x) \\
& -\int_{(0, t]} \int_{\mathbb{R}} x 1_{S}(x) d u \nu(d x)+\int_{(0, t]} \int_{\{|x| \leq 1\}} x d u \nu(d x) \\
= & \sigma \sqrt{h_{t}} W_{t}+\left[c \sqrt{h_{t}}+\int_{\{|x| \leq 1\}} x \nu(d x)-\int_{\mathbb{R}} x 1_{S}(x) \nu(d x)\right] t \\
& +\int_{(0, t]} \int_{\{|x| \leq 1\}} x \tilde{N}_{q}(d u d x)+\int_{(0, t]} \int_{\{|x|>1\}} x N_{q}(d u d x) \\
= & \sigma \sqrt{h_{t}} W_{t}+\left[c \sqrt{h_{t}}+\int_{\mathbb{R}} x\left(1_{\{|x| \leq 1\}}(x)-1_{S}(x)\right) \nu(d x)\right] t \\
& +\int_{(0, t]} \int_{\{|x| \leq 1\}} x \tilde{N}_{q}(d u d x)+\int_{(0, t]} \int_{\{|x|>1\}} x N_{q}(d u d x)
\end{aligned}
$$

We obtain the Lévy-Itô decomposition of $\left(X_{t}\right)$ associated with the truncation function $\tau(x):=x 1_{\{|x| \leq 1\}}$, and we derive the corresponding characteristics $\left(c^{\prime}, \sigma^{2} h_{t}, \nu\right)$, where

$$
c^{\prime}=c \sqrt{h_{t}}+\int_{\mathbb{R}} x\left(1_{\{|x| \leq 1\}}(x)-1_{S}(x)\right) \nu(d x) .
$$

We now come back to the proof of Proposition 5.2. From Proposition 2.1, we derive $\varphi^{\mathbb{Q}}(v)$

$$
\begin{aligned}
\varphi^{\mathbb{Q}}(v) & =\frac{\varphi^{\mathbb{P}}\left(\theta_{t}+v\right)}{\varphi^{\mathbb{P}}\left(\theta_{t}\right)} \\
& =\exp \left[\left(\theta_{t}+v-\theta_{t}\right) \widetilde{m}_{t}\right] \exp \left\{\left[\kappa\left(\left(\theta_{t}+v\right) \sqrt{h_{t}}\right)-\kappa\left(\theta_{t} \sqrt{h_{t}}\right)\right] t\right\} \\
& =\exp \left(v \widetilde{m}_{t}\right) \exp \left\{\left[\kappa\left(\left(\theta_{t}+v\right) \sqrt{h_{t}}\right)-\kappa\left(\theta_{t} \sqrt{h_{t}}\right)\right] t\right\} \\
& =\exp \left(v \widetilde{m}_{t}\right) \exp \left[\kappa^{\mathbb{Q}}\left(v \sqrt{h_{t}}\right) t\right]
\end{aligned}
$$


where

$$
\begin{aligned}
& \kappa^{\mathbb{Q}}\left(v \sqrt{h_{t}}\right)= \\
= & \kappa\left(\left(\theta_{t}+v\right) \sqrt{h_{t}}\right)-\kappa\left(\theta_{t} \sqrt{h_{t}}\right) \\
= & c\left(\theta_{t}+v\right) \sqrt{h_{t}}+\frac{\sigma^{2}}{2}\left(\theta_{t}+v\right)^{2} h_{t}+\int\left[e^{\left(\theta_{t}+v\right) \sqrt{h_{t}} x}-1-\tau(x)\left(\theta_{t}+v\right) \sqrt{h_{t}}\right] U(d x) \\
& -c \theta_{t} \sqrt{h_{t}}-\frac{\sigma^{2}}{2} \theta_{t}^{2} h_{t}-\int\left[e^{\theta_{t} \sqrt{h_{t}} x}-1-\tau(x) \theta_{t} \sqrt{h_{t}}\right] U(d x) \\
= & c v \sqrt{h_{t}}+\frac{\sigma^{2}}{2} v^{2} h_{t}+\sigma^{2} \theta_{t} h_{t} v+\int\left[e^{v \sqrt{h_{t}} x}-1-\tau(x) v \sqrt{h_{t}}\right] e^{\theta_{t} \sqrt{h_{t}} x} U(d x)+ \\
& \int\left(e^{\theta_{t} \sqrt{h_{t}} x}-1\right) \tau(x) \sqrt{h_{t}} v U(d x) \\
= & {\left[c+\sigma^{2} \theta_{t} \sqrt{h_{t}}+\int\left(e^{\theta_{t} \sqrt{h_{t}} x}-1\right) \tau(x) U(d x)\right] v \sqrt{h_{t}}+\frac{\sigma^{2}}{2}\left(v \sqrt{h_{t}}\right)^{2}+} \\
& \int\left(e^{v \sqrt{h_{t}} x}-1-\tau(x) v \sqrt{h_{t}}\right) e^{\theta_{t} \sqrt{h_{t}} x} U(d x) .
\end{aligned}
$$

Thus, under $\mathbb{Q},\left(z_{t}\right)_{t}$ is a Lévy process with triplet $\left(b, \sigma^{2}, U^{\mathbb{Q}}\right)$ where

$$
b=c+\sigma^{2} \theta_{t} \sqrt{h_{t}}+\int\left(e^{\theta_{t} \sqrt{h_{t}} z}-1\right) \tau(z) U(d z)
$$

and

$$
U^{\mathbb{Q}}(d x)=e^{\theta_{t} \sqrt{h_{t}} x} U(d x) .
$$

Using Lemma 5.5, $X_{t}=\sqrt{h_{t}} z_{t}$ is a Lévy process with triplet $\left(c^{\prime}, \sigma^{2} h_{t}, \nu^{\mathbb{Q}}\right)$ where

$$
c^{\prime}=c^{\mathbb{Q}}=b \sqrt{h_{t}}+\int_{\mathbb{R}} x\left(1_{\{|x| \leq 1\}}(x)-1_{S}(x)\right) \nu^{\mathbb{Q}}(d x),
$$

and

$$
\nu^{\mathbb{Q}}(d x)=U^{\mathbb{Q}}\left(\frac{d x}{\sqrt{h_{t}}}\right)=e^{\theta_{t} x} U\left(\frac{d x}{\sqrt{h_{t}}}\right)
$$

\section{References}

[1] Aît-Sahalia Y., and Lo A., 1998. Nonparametric estimation of state-price densities implicit in financial asset prices. Journal of Finance, 53, 499-547.

[2] Aît-Sahalia Y., and Lo A., 2000. Nonparametric risk management and implied risk aversion. Journal of Econometrics, 94, 9-51.

[3] Badescu A., and Kulperger R., 2007. GARCH option pricing: A semiparametric approach. Insurance: Mathematics and Economics, 43(1), 69-84. 
[4] Bakshi G., and Kapadia N., 2003. "Delta-Hedged Gains and the Negative Market Volatility Premium.” Review of Financial Studies, 16, 527-566.

[5] Barone-Adesi G., Engle R., Mancini L., 2008. A GARCH Option Pricing Model with Filtered Historical Simulation. Review of Financial Studies, 21(3), 1223-1258.

[6] Bates D., 1996. Jumps and stochastic volatility: exchange rate processes implicit in Deutsche mark options. Review of Financial Studies 9, 69-107.

[7] Black F., Scholes M., 1973. The pricing of options and corporate liabilities. Journal of Political Economy, 81, 637-659.

[8] Bollen N., and Whaley R., 2003. "What Determines the Shape of Implied Volatility Functions.” Journal of Finance, forthcoming.

[9] Bouchaud J.P. and Potters M., 2003. Theory of Financial Risk and Derivative Pricing. Cambridge University Press.

[10] Brown D.P. and Jackwerth J., 2001. The Pricing Kernel Puzzle: Reconciling Index Option Data and Economic Theory. Working Paper University of Wisconsin.

[11] Chacko G. , Viceira Luis M., 2003. Spectral GMM estimation of continuous-time processes. Journal of Econometrics, Elsevier, 116, 259-292.

[12] Chevallier J., Ielpo F., and Mercier L., 2008. 'Risk Aversion and Institutional Information Disclosure on the European Carbon Market: a Case-Study of the 2006 Compliance Event'. Energy Policy 37(1), 15-28.

[13] Christoffersen P., Heston S., Jacobs K., 2006. Option valuation with conditional skewness, Journal of Econometrics, 131, 253-284.

[14] Christoffersen P., Heston S., Jacobs K., 2011. A GARCH Option Model with Variance-Dependent Pricing Kernel.

[15] Chorro C., Guégan D., Ielpo F., 2010a. Martingalized Historical approach for Option Pricing. Finance Research letters, 7, 24-28.

[16] Chorro C., Guégan D., Ielpo F., 2012. Option pricing for GARCH-type models with generalized hyperbolic innovations. To appear in Quantitative Finance.

[17] Coval J., and Shumway T., 2001. "Expected Option Returns." Journal of Finance $56,983-1009$.

[18] Ding Z., Granger C.W.J. and Engle R.F., 1993. A long memory property of stock market returns and a new model. Journal of Empirical Finance 1, 83-106.

[19] Duan J.C., Ritchken P., Sun Z., 2007. Jump Starting GARCH: Pricing Options with Jumps in Returns and Volatilities. Risk Management Institute WP 07-35, National University of Singapore.

[20] Duan J.C., Simonato J.G., 1998. Empirical Martingale Simulation for asset Prices. Management Science, 44(9), 1218-1233. 
[21] Duffie D., Pan J., and Singleton K., 2000. Transform analysis and asset pricing for affine jump-diffusion. Econometrica, 68(6), 1343-1376.

[22] Eberlein E., Prause K., 2002. The generalized hyperbolic model: financial derivatives and risk measures. In: H. Geman, D. Madan, S. Pliska, T. Vorst (Eds.), Mathematical Finance-Bachelier Congress 2000, 245-267, Springer Verlag, Paris.

[23] Elliott R. and Madan D., 1998. A Discrete Time Equivalent Martingale Measure. Mathematical Finance, 2(8), 127-152.

[24] Fujiwara T. and Miyahara Y., 2003. The minimal entropy martingale measures for geometric Lévy processes. Finance Stoch., 7(4), 509-531.

[25] Gerber H.U. and Shiu S.W., 1994a. Martingale Approach to Pricing Perpetual American Options. In: Proceedings of the 4th AFIR International Colloqium, Transaction of Society of Actuaries: Orlando, 659-689.

[26] Gerber H.U. and Shiu S.W., 1994b. Option Pricing by Esscher Transforms. Transaction of Society of Actuaries, 46, 99-191.

[27] Glosten L.R., Jagannathan R. and Runkle D.E., 1993. On the Relation Between the Expected Value and the Volatility of the Nominal Excess Returns on Stocks. J. Financ., 48, 1791-1801.

[28] Gourieroux C., Monfort A. and Trognon A., 1984a. Pseudo maximum likelihood methods: Applications to Poisson models. Econometrica, 52(3), 701-720.

[29] Heston S., 1993. A closed form solution for options with stochastic volatility with applications to bond and currency options. Review of Financial Studies, 6, 327 343.

[30] Jackwerth J., 2000, "Recovering Risk Aversion from Option Prices and Realized Returns." Review of Financial Studies 13, 433-451.

[31] Merton R.C., 1973. Theory of rational option pricing. Bell Journal of Economics and Management Science, 4, 141-183.

[32] Merton R. C., 1976. Option Pricing When Underlying Stock Returns are Discontinuous. Journal of Financial Economics, 3, 125-144.

[33] Michael R. , Maria., 2005. Estimation of Jump-Diffusion Process vis Empirical Characteristic Function, FAME Research Paper Series 150. International Center for Financial Asset Management and Engineering, Genève.

[34] Miyahara Y. , Moriwaki N. , 2009. Option Pricing Based on Geometric Stable Processes and Minimal Entropy Martingale Measures. In "Recent Advances in Financial Engineering”, World Sci. Publ., 119-133.

[35] Nelson D.B., 1991. Conditional heteroskedasticity in asset returns: A new approach. Econometrica, 59, 347-370. 
[36] Rosenberg, Joshua V. , Engle, Robert F., 2002. Empirical pricing kernels. Journal of Financial Economics, Elsevier, 64(3), 341-372.

[37] Schoutens W., 2003. Lévy Processes in Finance: Pricing Financial Derivatives. Wiley. 


\section{Tables and Figures}

\begin{tabular}{|c|c|c|c|}
\multicolumn{5}{|c|}{ CAC 40 } \\
\hline \hline Number of observations & Mean & Minimum & Maximum \\
\hline 5052 & 0.036 & -0.095 & 0.106 \\
\hline \hline Median & Standard Deviation & Skewness & Kurtosis \\
\hline $3.375 \mathrm{e}-004$ & 0.222 & -0.044 & 7.703 \\
\hline \hline
\end{tabular}

\section{S\&P 500}

\begin{tabular}{|c|c|c|c|}
\hline \hline Number of observations & Mean & Minimum & Maximum \\
\hline 5040 & 0.061 & -0.095 & 0.109 \\
\hline \hline Median & Standard Deviation & Skewness & Kurtosis \\
\hline $5.230 \mathrm{e}-004$ & 0.190 & -0.203 & 9.147 \\
\hline \hline
\end{tabular}

Table 1: Descriptive statistics for the CAC 40 and the S\&P 500 log returns. This table presents the descriptive statistics for the CAC 40 and the S\&P 500 log returns from March 1, 1990 to March 1, 2010.

\begin{tabular}{|l|c|c|}
\hline \hline & CAC 40 & S\&P 500 \\
\hline \hline KS p-value for Normal-EGARCH & 0.016 & $1.288 \mathrm{e}-06$ \\
KS p-value for Normal-GJR & 0.013 & $5.058 \mathrm{e}-07$ \\
KS p-value for Normal-APARCH & 0.017 & $2.056 \mathrm{e}-06$ \\
\hline KS p-value for NIG-EGARCH & 0.738 & 0.477 \\
KS p-value for NIG-GJR & 0.721 & 0.532 \\
KS p-value for NIG-APARCH & 0.671 & 0.454 \\
\hline KS p-value for Model (2)-EGARCH & 0.457 & 0.827 \\
KS p-value for Model (2)-GJR & 0.635 & 0.815 \\
KS p-value for Model (2)-APARCH & 0.584 & 0.464 \\
\hline KS p-value for Model (3)-EGARCH & 0.716 & 0.610 \\
KS p-value for Model (3)-GJR & 0.699 & 0.633 \\
KS p-value for Model (3)-APARCH & 0.648 & 0.571 \\
\hline \hline AD p-value for Normal-EGARCH & 0.030 & 0.040 \\
AD p-value for Normal-GJR & 0.010 & 0.010 \\
AD p-value for Normal-APARCH & 0.010 & 0.020 \\
\hline AD p-value for NIG-EGARCH & 0.760 & 0.630 \\
AD p-value for NIG-GJR & 0.470 & 0.520 \\
AD p-value for NIG-APARCH & 0.460 & 0.410 \\
\hline AD p-value for Model (2)-EGARCH & 0.430 & 0.700 \\
AD p-value for Model (2)-GJR & 0.840 & 0.660 \\
AD p-value for Model (2)-APARCH & 0.840 & 0.320 \\
\hline AD p-value for Model (3)-EGARCH & 0.680 & 0.530 \\
AD p-value for Model (3)-GJR & 0.660 & 0.490 \\
AD p-value for Model (3)-APARCH & 0.450 & 0.370 \\
\hline \hline
\end{tabular}

Table 2: Kolmogorov-Smirnov, Anderson-Darling adequation tests

This table presents the test statistics testing the adequation of the residuals of the models EGARCH, GJR and APARCH respectively with Normal, NIG and Poisson distributions as defined in (1)-(7) to a data set of daily log returns of the French CAC 40 and of the S\&P 500 from March 1, 1990 to March 1, 2010. 
CAC 40

\begin{tabular}{|c|c|c|c|c|c|}
\hline \hline Maturity/Moneyness & $<0.8$ & {$[.8-.9]$} & {$[.9-1]$} & {$[1-1.1]$} & $>1.1$ \\
\hline \hline 7 days $\leq$ Maturity $\leq 30$ days & 1629 & 3342 & 3100 & 687 & 11 \\
\hline 3 months $\leq$ Maturity $\leq 6$ months & 1140 & 2135 & 2333 & 1784 & 579 \\
\hline Maturity $>6$ months & 5256 & 9176 & 9328 & 8289 & 3503 \\
\hline \hline
\end{tabular}

S\&P 500

\begin{tabular}{|c|c|c|c|c|c|}
\hline \hline Maturity/Moneyness & $<0.8$ & {$[.8-.9]$} & {$[.9-1]$} & {$[1-1.1]$} & $>1.1$ \\
\hline \hline 7 days $\leq$ Maturity $\leq 30$ days & 1010 & 1937 & 2066 & 1839 & 253 \\
\hline 3 months $\leq$ Maturity $\leq 6$ months & 876 & 1776 & 1968 & 1976 & 333 \\
\hline Maturity $>6$ months & 1398 & 4280 & 6814 & 5505 & 1369 \\
\hline \hline
\end{tabular}

Table 3: Descriptive statistics for the option data sets used in the paper. The option data sets start on December 6, 2006 and end on October 26, 2007.

\begin{tabular}{|c|c|c|c|c|c|}
\hline \hline Index & EGARCH parameters & $\alpha_{0}$ & $\beta_{1}$ & $\alpha_{1}$ & $\gamma_{1}$ \\
\hline \hline CAC 40 & Mean & $-1.15 \mathrm{e}-01$ & 0.987 & 0.100 & -0.059 \\
& Stand.Dev & $6.93 \mathrm{e}-03$ & 0.0008 & 0.009 & 0.003 \\
\hline \hline S\&P 500 & Mean & -0.148 & 0.984 & 0.111 & -0.089 \\
& Stand.Dev & 0.009 & $8.86 \mathrm{e}-4$ & 0.002 & 0.004 \\
\hline \hline
\end{tabular}

Table 4: Estimated parameters for the EGARCH model, using a QuasiMaximum Likelihood Estimation.

This table presents the estimated parameters with their standard deviation for the EGARCH model by selecting a subsample containing 4000 working days and ending on the date $t$ between December 6, 2006 and October 26, 2007.

\begin{tabular}{|c|c|c|c|c|c|}
\hline \hline Index & GJR parameters & $\omega$ & $\beta$ & $\gamma$ & $\delta$ \\
\hline \hline CAC 40 & Mean & $2.07 \mathrm{e}-06$ & 0.934 & 0.016 & 0.072 \\
& Stand.Dev & $3.04 \mathrm{e}-07$ & 0.004 & 0.001 & 0.005 \\
\hline \hline S\&P 500 & Mean & $9.463 \mathrm{e}-07$ & 0.934 & 0.003 & 0.104 \\
& Stand.Dev & $5.307 \mathrm{e}-08$ & 0.002 & 0.003 & 0.005 \\
\hline \hline
\end{tabular}

Table 5: Estimated parameters for the GARCH-GJR model, using a QuasiMaximum Likelihood Estimation.

This table presents the estimated parameters with their standard deviation for the GARCH-GJR model by selecting a subsample containing 4000 working days and ending on the date $t$ between December 6, 2006 and October 26, 2007. 


\begin{tabular}{|c|c|c|c|c|c|c|}
\hline \hline Index & APARCH parameters & $\omega$ & $a_{1}$ & $\gamma_{1}$ & $b_{1}$ & $\delta$ \\
\hline \hline CAC 40 & Mean & $6.91 \mathrm{e}-05$ & 0.053 & 0.571 & 0.941 & 1.220 \\
& Stand.Dev & $2.07 \mathrm{e}-05$ & 0.003 & 0.024 & 0.004 & 0.078 \\
\hline \hline S\&P 500 & Mean & $8.745 \mathrm{e}-05$ & 0.057 & 0.829 & 0.939 & 1.100 \\
& Stand.Dev & $1.890 \mathrm{e}-05$ & 0.002 & 0.053 & 0.001 & 0.044 \\
\hline \hline
\end{tabular}

Table 6: Estimated parameters for the APARCH model, using a Quasi-Maximum Likelihood Estimation.

This table presents the estimated parameters with their standard deviation for the APARCH model by selecting a subsample containing 4000 working days and ending on the date $t$ between December 6, 2006 and October 26, 2007.

\begin{tabular}{|c|c|c|c|c|c|c|}
\hline \hline Index & Models & & $\alpha$ & $\beta$ & $\delta$ & $\mu$ \\
\hline \hline CAC 40 & NIG-EGARCH & Mean & 2.484 & -0.397 & 2.371 & 0.382 \\
& & Stand.Dev & 0.229 & 0.065 & 0.215 & 0.064 \\
& \multirow{2}{*}{ NIG-GJR } & Mean & 2.487 & -0.412 & 2.369 & 0.396 \\
& & Stand.Dev & 0.212 & 0.059 & 0.198 & 0.056 \\
& \multirow{2}{*}{ NIG-APARCH } & Mean & 2.492 & -0.396 & 2.366 & 0.381 \\
& & Stand.Dev & 0.222 & 0.062 & 0.209 & 0.060 \\
\hline \hline S\&P 500 & \multirow{2}{*}{ NIG-EGARCH } & Mean & 1.785 & -0.175 & 1.749 & 0.174 \\
& & Stand.Dev & 0.170 & 0.069 & 0.149 & 0.064 \\
& \multirow{2}{*}{ NIG-GJR } & Mean & 1.785 & -0.208 & 1.738 & 0.202 \\
& & Stand.Dev & 0.171 & 0.071 & 0.147 & 0.066 \\
& \multirow{2}{*}{ NIG-APARCH } & Mean & 1.788 & -0.179 & 1.752 & 0.180 \\
& & Stand.Dev & 0.171 & 0.069 & 0.149 & 0.065 \\
\hline \hline
\end{tabular}

Table 7: Estimated parameters for the NIG distribution, using the previous volatility dynamics, by MLE.

This table presents the estimated parameters for the NIG distribution using the 2 stages estimation procedure and 4000 log-returns. NIG-GARCH means the model (1) with NIG innovation associated with its GARCH model.

\begin{tabular}{|c|c|c|c|c|c|}
\hline Index & Models & & $\lambda$ & $\mu$ & $\overline{\sigma^{2}}$ \\
\hline \multirow[t]{6}{*}{ CAC 40} & Model (2)-EGA & Mean & 0.141 & 0.004 & 0.033 \\
\hline & \multirow{3}{*}{ Model (2)-GJR } & Stand.Dev & 0.000 & 0.020 & 0.016 \\
\hline & & Mean & 0.143 & 0.087 & 0.027 \\
\hline & & Stand.Dev & 0.009 & 0.026 & 0.045 \\
\hline & \multirow{2}{*}{ Model (2)-APARCH } & Mean & 0.139 & 0.059 & 0.038 \\
\hline & & Stand.Dev & 0.000 & 0.022 & 0.015 \\
\hline \multirow[t]{6}{*}{ S\&P 500 } & \multirow[t]{2}{*}{ Model (2)-EGARCH } & Mean & 0.15 & 0.015 & $\overline{0.009}$ \\
\hline & & Stand.Dev & 0.049 & 0.005 & 0.004 \\
\hline & \multirow{2}{*}{ Model (2)-GJR } & Mean & 0.148 & -0.008 & 0.010 \\
\hline & & Stand.Dev & 0.018 & 0.004 & 0.007 \\
\hline & \multirow[t]{2}{*}{ Model (2)-APARCH } & Mean & 0.152 & 0.027 & 0.008 \\
\hline & & Stand.Dev & 0.024 & 0.004 & 0.004 \\
\hline
\end{tabular}

Table 8: Estimated parameters for the Poisson and normal distributions, using the previous volatility dynamics, by MLE.

This table presents the average estimated parameters for the Poisson and normal distributions using the 2 stages estimation procedure and 4000 log-returns. Model (2)GARCH means the model (2) associated with its GARCH model. 


\begin{tabular}{|c|c|c|c|c|c|}
\hline \hline Index & Models & & $\lambda$ & $\mu$ & $\sigma$ \\
\hline \hline CAC 40 & Model (3)-EGARCH & Mean & 0.057 & -0.314 & 0.911 \\
& & Stand.Dev & 0.024 & 0.216 & 0.171 \\
& Model (3)-GJR & Mean & 0.067 & -0.258 & 0.821 \\
& & Stand.Dev & 0.031 & 0.188 & 0.158 \\
& Model (3)-APARCH & Mean & 0.066 & -0.251 & 0.825 \\
& & Stand.Dev & 0.031 & 0.189 & 0.160 \\
\hline \hline S\&P 500 & Model (3)-EGARCH & Mean & 0.064 & 0.028 & 0.009 \\
& & Stand.Dev & 0.028 & 0.034 & 0.006 \\
& \multirow{2}{*}{ Model (3)-GJR } & Mean & 0.057 & $-1.681 \mathrm{e}-05$ & $9.613 \mathrm{e}-03$ \\
& & Stand.Dev & 0.038 & 0.001 & 0.002 \\
& \multirow{2}{*}{ Model (3)-APARCH } & Mean & 0.072 & 0.043 & 0.009 \\
& & Stand.Dev & 0.041 & 0.027 & 0.005 \\
\hline \hline
\end{tabular}

Table 9: Estimated parameters for the Poisson and normal distributions, using the previous volatility dynamics, by MLE.

This table presents the estimated parameters for the Poisson and normal distributions using the 2 stages estimation procedure and 4000 log-returns. Model (3)-GARCH means the model (3) associated with its GARCH model.

\begin{tabular}{lccccc}
\multicolumn{7}{c}{7 days $\leq$ Maturity $\leq 30$ days } \\
\hline \hline Moneyness & $<0.8$ & {$[.8-.9]$} & {$[.9-1]$} & {$[1-1.1]$} & $>1.1$ \\
\hline \hline Nig-EGARCH Ess & 0.051 & 0.079 & 0.137 & 2.105 & 1.971 \\
Nig-GJR Ess & 0.051 & 0.079 & 0.136 & 2.204 & 2.738 \\
Nig-APARCH Ess & 0.050 & 0.077 & 0.107 & 2.576 & 3.056 \\
\hline Nig-EGARCH MEMM & 0.046 & 0.071 & 0.129 & 0.365 & 1.914 \\
Nig-GJR MEMM & 0.046 & 0.071 & 0.130 & 0.367 & 3.600 \\
Nig-APARCH MEMM & 0.044 & 0.067 & 0.099 & 2.235 & 2.835 \\
\hline Model (2)-EGARCH Ess & 0.053 & 0.073 & 0.186 & 0.371 & 2.215 \\
Model (2)-GJR Ess & 0.054 & 0.073 & 0.187 & 0.373 & 2.842 \\
Model (2)-APARCH Ess & 0.051 & 0.071 & 0.095 & 1.568 & 5.021 \\
\hline Model (2)-EGARCH MEMM & 0.049 & 0.068 & 0.154 & 0.486 & 3.482 \\
Model (2)-GJR MEMM & 0.049 & 0.068 & 0.154 & 0.482 & 4.037 \\
Model (2)-APARCH MEMM & 0.043 & 0.064 & $\mathbf{0 . 0 9 1}$ & $\mathbf{0 . 3 5 2}$ & $\mathbf{1 . 8 9 8}$ \\
\hline Model (3)-EGARCH Ess & 0.097 & 0.127 & 0.165 & 0.881 & 3.014 \\
Model (3)-GJR Ess & 0.096 & 0.126 & 0.166 & 0.882 & 3.020 \\
Model (3)-APARCH Ess & $\mathbf{0 . 0 4 0}$ & $\mathbf{0 . 0 4 6}$ & 0.190 & 0.981 & 4.261 \\
\hline Model (3)-EGARCH MEMM & 0.047 & 0.052 & 0.186 & 0.949 & 4.029 \\
Model (3)-GJR MEMM & 0.080 & 0.084 & 0.101 & 0.947 & 4.013 \\
Model (3)-APARCH MEMM & 0.044 & 0.121 & 0.303 & 2.896 & 6.242 \\
\hline \hline
\end{tabular}


3 months $\leq$ Maturity $\leq 6$ months

\begin{tabular}{lccccc}
\hline \hline Moneyness & $<0.8$ & {$[.8-.9]$} & {$[.9-1]$} & {$[1-1.1]$} & $>1.1$ \\
\hline \hline Nig-EGARCH Ess & 0.040 & 0.084 & 0.279 & $\mathbf{0 . 5 2 1}$ & $\mathbf{0 . 6 1 4}$ \\
Nig-GJR Ess & 0.040 & 0.084 & 0.283 & 0.526 & 0.619 \\
Nig-APARCH Ess & 0.061 & 0.102 & 0.402 & 0.868 & 4.125 \\
\hline Nig-EGARCH MEMM & 0.057 & 0.115 & 0.752 & 3.084 & 3.389 \\
Nig-GJR MEMM & 0.056 & 0.114 & 0.755 & 3.052 & 3.342 \\
Nig-APARCH MEMM & 0.081 & 0.181 & 0.791 & 0.881 & 4.051 \\
\hline Model (2)-EGARCH Ess & $\mathbf{0 . 0 3 2}$ & $\mathbf{0 . 0 7 1}$ & 0.362 & 0.765 & 2.562 \\
Model (2)-GJR Ess & 0.033 & 0.073 & 0.365 & 0.776 & 2.684 \\
Model (2)-APARCH Ess & 0.061 & 0.122 & 0.756 & 0.865 & 4.103 \\
\hline Model (2)-EGARCH MEMM & 0.052 & 0.082 & 0.385 & 0.796 & 2.652 \\
Model (2)-GJR MEMM & 0.054 & 0.084 & 0.384 & 0.798 & 2.513 \\
Model (2)-APARCH MEMM & 0.070 & 0.192 & 0.825 & 1.004 & 4.301 \\
\hline Model (3)-EGARCH Ess & 0.070 & 0.129 & 0.269 & 0.960 & 1.265 \\
Model (3)-GJR Ess & 0.071 & 0.130 & 0.276 & 0.971 & 1.389 \\
Model (3)-APARCH Ess & 0.047 & 0.073 & 0.213 & 0.964 & 1.025 \\
\hline Model (3)-EGARCH MEMM & 0.081 & 0.084 & $\mathbf{0 . 0 9 9}$ & 0.941 & 1.952 \\
Model (3)-GJR MEMM & 0.157 & 0.158 & 0.124 & 0.939 & 1.941 \\
Model (3)-APARCH MEMM & 0.055 & 0.127 & 0.237 & 2.594 & 1.022 \\
\hline \hline
\end{tabular}


Maturity $>6$ months

\begin{tabular}{lccccc}
\hline \hline Moneyness & $<0.8$ & {$[.8-.9]$} & {$[.9-1]$} & {$[1-1.1]$} & $>1.1$ \\
\hline \hline Nig-EGARCH Ess & 0.056 & 0.081 & 0.120 & $\mathbf{0 . 2 6 8}$ & $\mathbf{0 . 3 1 8}$ \\
Nig-GJR Ess & 0.057 & 0.082 & 0.121 & 0.269 & 0.321 \\
Nig-APARCH Ess & 0.058 & 0.074 & 0.142 & 0.302 & 0.351 \\
\hline Nig-EGARCH MEMM & 0.051 & 0.068 & 0.249 & 0.436 & 0.454 \\
Nig-GJR MEMM & 0.051 & 0.067 & 0.245 & 0.445 & 0.466 \\
Nig-APARCH MEMM & 0.065 & 0.095 & 0.291 & 0.475 & 0.501 \\
\hline Model (2)-EGARCH Ess & $\mathbf{0 . 0 5 0}$ & $\mathbf{0 . 0 6 6}$ & $\mathbf{0 . 1 1 1}$ & 0.302 & 0.321 \\
Model (2)-GJR Ess & 0.052 & 0.067 & 0.118 & 0.321 & 0.329 \\
Model (2)-APARCH Ess & 0.062 & 0.083 & 0.136 & 0.391 & 4.011 \\
\hline Model (2)-EGARCH MEMM & 0.521 & 0.071 & 0.224 & 0.201 & 0.405 \\
Model (2)-GJR MEMM & 0.526 & 0.072 & 0.227 & 0.211 & 0.402 \\
Model (2)-APARCH MEMM & 0.068 & 0.076 & 0.381 & 0.445 & 4.213 \\
\hline Model (3)-EGARCH Ess & 0.053 & 0.375 & 0.230 & 0.902 & 0.996 \\
Model (3)-GJR Ess & 0.054 & 0.376 & 0.229 & 0.907 & 0.998 \\
Model (3)-APARCH Ess & 0.090 & 0.076 & 0.161 & 0.818 & 0.824 \\
\hline Model (3)-EGARCH MEMM & 0.661 & 0.679 & 0.548 & 0.599 & 0.991 \\
Model (3)-GJR MEMM & 1.531 & 1.564 & 1.363 & 0.864 & 0.983 \\
Model (3)-APARCH MEMM & 0.552 & 0.963 & 0.941 & 0.921 & 1.396 \\
\hline \hline
\end{tabular}

Table 10: Absolute Average Relative Pricing Errors (AARPE) for the CAC 40 French index.

These tables present the AARPE using the NIG-EGARCH, NIG-GJR, NIG-APARCH, Model (2)-EGARCH, Model (2)-GJR, Model (2)-APARCH, Model (3)-EGARCH, Model (3)-GJR and Model (3)-APARCH under an exponential affine stochastic discount factor (also known as Esscher transform ESS) or under a minimal entropy martingal measure (MEMM). For example, NIG-EGARCH ESS means we use the model (1) with NIG innovation and EGARCH volatility model associated with an exponential affine stochastic discount factor. We put the minimal errors in bold face. 
7 days $\leq$ Maturity $\leq 30$ days

\begin{tabular}{lccccc}
\hline \hline Moneyness & $<0.8$ & {$[.8-.9]$} & {$[.9-1]$} & {$[1-1.1]$} & $>1.1$ \\
\hline \hline Nig-EGARCH Ess & 0.109 & 0.116 & 0.198 & 11.290 & 1.031 \\
Nig-GJR Ess & 0.109 & 0.117 & 0.195 & 11.357 & 1.298 \\
Nig-APARCH Ess & 0.109 & 0.116 & 0.197 & 11.335 & 1.327 \\
\hline Nig-EGARCH MEMM & 0.111 & 0.120 & 0.216 & 11.481 & 1.131 \\
Nig-GJR MEMM & 0.112 & 0.122 & 0.214 & 11.954 & 1.511 \\
Nig-APARCH MEMM & 0.109 & 0.118 & 0.204 & 11.067 & 1.263 \\
\hline Model (2)-EGARCH Ess & $\mathbf{0 . 1 0 5}$ & $\mathbf{0 . 1 1 2}$ & 0.130 & 0.988 & 1.116 \\
Model (2)-GJR Ess & 0.106 & 0.114 & 0.133 & 0.992 & 1.123 \\
Model (2)-APARCH Ess & 0.105 & 0.113 & 0.132 & 0.989 & 1.119 \\
\hline Model (2)-EGARCH MEMM & 0.112 & 0.119 & $\mathbf{0 . 1 2 2}$ & $\mathbf{0 . 9 8 0}$ & $\mathbf{1 . 0 1 6}$ \\
Model (2)-GJR MEMM & 0.108 & 0.115 & 0.123 & 0.983 & 1.213 \\
Model (2)-APARCH MEMM & 0.107 & 0.114 & 0.126 & 0.986 & 1.222 \\
\hline Model (3)-EGARCH Ess & 0.106 & 0.112 & 0.130 & 0.990 & 1.097 \\
Model (3)-GJR Ess & 0.107 & 0.117 & 0.138 & 0.995 & 1.236 \\
Model (3)-APARCH Ess & 0.107 & 0.114 & 0.131 & 0.991 & 1.119 \\
\hline Model (3)-EGARCH MEMM & 0.111 & 0.119 & 0.122 & 0.982 & 1.325 \\
Model (3)-GJR MEMM & 0.109 & 0.116 & 0.123 & 0.985 & 1.237 \\
Model (3)-APARCH MEMM & 0.107 & 0.114 & 0.126 & 0.988 & 1.215 \\
\hline \hline
\end{tabular}

3 months $<$ Maturity $<6$ months

\begin{tabular}{lccccc}
\hline \hline Moneyness & $<0.8$ & {$[.8-.9]$} & {$[.9-1]$} & {$[1-1.1]$} & $>1.1$ \\
\hline \hline Nig-EGARCH Ess & 0.172 & 0.157 & 0.204 & 1.648 & 6.618 \\
Nig-GJR Ess & 0.175 & 0.163 & 0.203 & 1.560 & 6.657 \\
Nig-APARCH Ess & 0.174 & 0.160 & 0.201 & 1.541 & 6.486 \\
\hline Nig-EGARCH MEMM & 0.185 & 0.181 & 0.253 & 1.881 & 7.629 \\
Nig-GJR MEMM & 0.179 & 0.171 & 0.236 & 1.735 & 6.459 \\
Nig-APARCH MEMM & 0.175 & 0.167 & 0.215 & 1.541 & 6.338 \\
\hline Model (2)-EGARCH Ess & $\mathbf{0 . 1 5 4}$ & $\mathbf{0 . 1 2 1}$ & 0.174 & 0.940 & 1.229 \\
Model (2)-GJR Ess & 0.155 & 0.123 & 0.177 & 0.945 & 1.459 \\
Model (2)-APARCH Ess & 0.154 & 0.121 & 0.175 & 0.941 & 1.332 \\
\hline Model (2)-EGARCH MEMM & 0.183 & 0.157 & $\mathbf{0 . 1 2 5}$ & 0.868 & 1.113 \\
Model (2)-GJR MEMM & 0.171 & 0.143 & 0.132 & 0.896 & 1.215 \\
Model (2)-APARCH MEMM & 0.163 & 0.132 & 0.147 & 0.918 & 1.203 \\
\hline Model (3)-EGARCH Ess & 0.154 & 0.223 & 0.158 & $\mathbf{0 . 7 4 8}$ & $\mathbf{0 . 9 9 8}$ \\
Model (3)-GJR Ess & 0.154 & 0.125 & 0.177 & 0.942 & 1.119 \\
Model (3)-APARCH Ess & 0.156 & 0.124 & 0.161 & 0.897 & 1.109 \\
\hline Model (3)-EGARCH MEMM & 0.183 & 0.157 & 0.125 & 0.871 & 1.205 \\
Model (3)-GJR MEMM & 0.171 & 0.142 & 0.133 & 0.901 & 1.310 \\
Model (3)-APARCH MEMM & 0.180 & 0.155 & 0.127 & 0.882 & 1.212 \\
\hline \hline
\end{tabular}


Maturity $>6$ months

\begin{tabular}{lccccc}
\hline \hline Moneyness & $<0.8$ & {$[.8-.9]$} & {$[.9-1]$} & {$[1-1.1]$} & $>1.1$ \\
\hline \hline Nig-EGARCH Ess & 0.409 & 0.362 & 0.359 & 0.685 & 2.521 \\
Nig-GJR Ess & 0.431 & 0.391 & 0.386 & 0.675 & 2.348 \\
Nig-APARCH Ess & 0.413 & 0.366 & 0.351 & 0.623 & 2.242 \\
\hline Nig-EGARCH MEMM & 0.446 & 0.421 & 0.449 & 0.837 & 2.899 \\
Nig-GJR MEMM & 0.452 & 0.426 & 0.451 & 0.841 & 2.905 \\
Nig-APARCH MEMM & 0.411 & 0.374 & 0.372 & 0.648 & 2.192 \\
\hline Model (2)-EGARCH Ess & $\mathbf{0 . 3 3 1}$ & $\mathbf{0 . 2 2 4}$ & 0.158 & 0.745 & 0.998 \\
Model (2)-GJR Ess & 0.332 & 0.226 & 0.161 & 0.748 & 1.113 \\
Model (2)-APARCH Ess & 0.332 & 0.224 & 0.159 & 0.746 & 1.008 \\
\hline Model (2)-EGARCH MEMM & 0.438 & 0.361 & 0.230 & $\mathbf{0 . 5 1 4}$ & $\mathbf{0 . 9 3 0}$ \\
Model (2)-GJR MEMM & 0.398 & 0.307 & 0.177 & 0.588 & 0.970 \\
Model (2)-APARCH MEMM & 0.366 & 0.266 & $\mathbf{0 . 1 5 5}$ & 0.662 & 0.989 \\
\hline Model (3)-EGARCH Ess & 0.334 & 0.225 & 0.159 & 0.849 & 1.229 \\
Model (3)-GJR Ess & 0.337 & 0.228 & 0.167 & 0.859 & 1.325 \\
Model (3)-APARCH Ess & 0.336 & 0.226 & 0.165 & 0.857 & 1.319 \\
\hline Model (3)-EGARCH MEMM & 0.439 & 0.361 & 0.230 & 0.518 & 0.935 \\
Model (3)-GJR MEMM & 0.397 & 0.306 & 0.176 & 0.593 & 0.972 \\
Model (3)-APARCH MEMM & 0.419 & 0.311 & 0.180 & 0.590 & 0.975 \\
\hline \hline
\end{tabular}

Table 11: Absolute Average Relative Pricing Errors (AARPE) for the S\&P 500 index.

These tables present the AARPE using the NIG-EGARCH, NIG-GJR, NIG-APARCH, Model (2)-EGARCH, Model (2)-GJR, Model (2)-APARCH, Model (3)-EGARCH, Model (3)-GJR and Model (3)-APARCH under an exponential affine stochastic discount factor (also known as Esscher transform ESS) or under a minimal entropy martingal measure (MEMM). For example, NIG-EGARCH ESS means we use the model (1) with NIG innovation and EGARCH volatility model associated with an exponential affine stochastic discount factor. We put the minimal errors in bold face. 

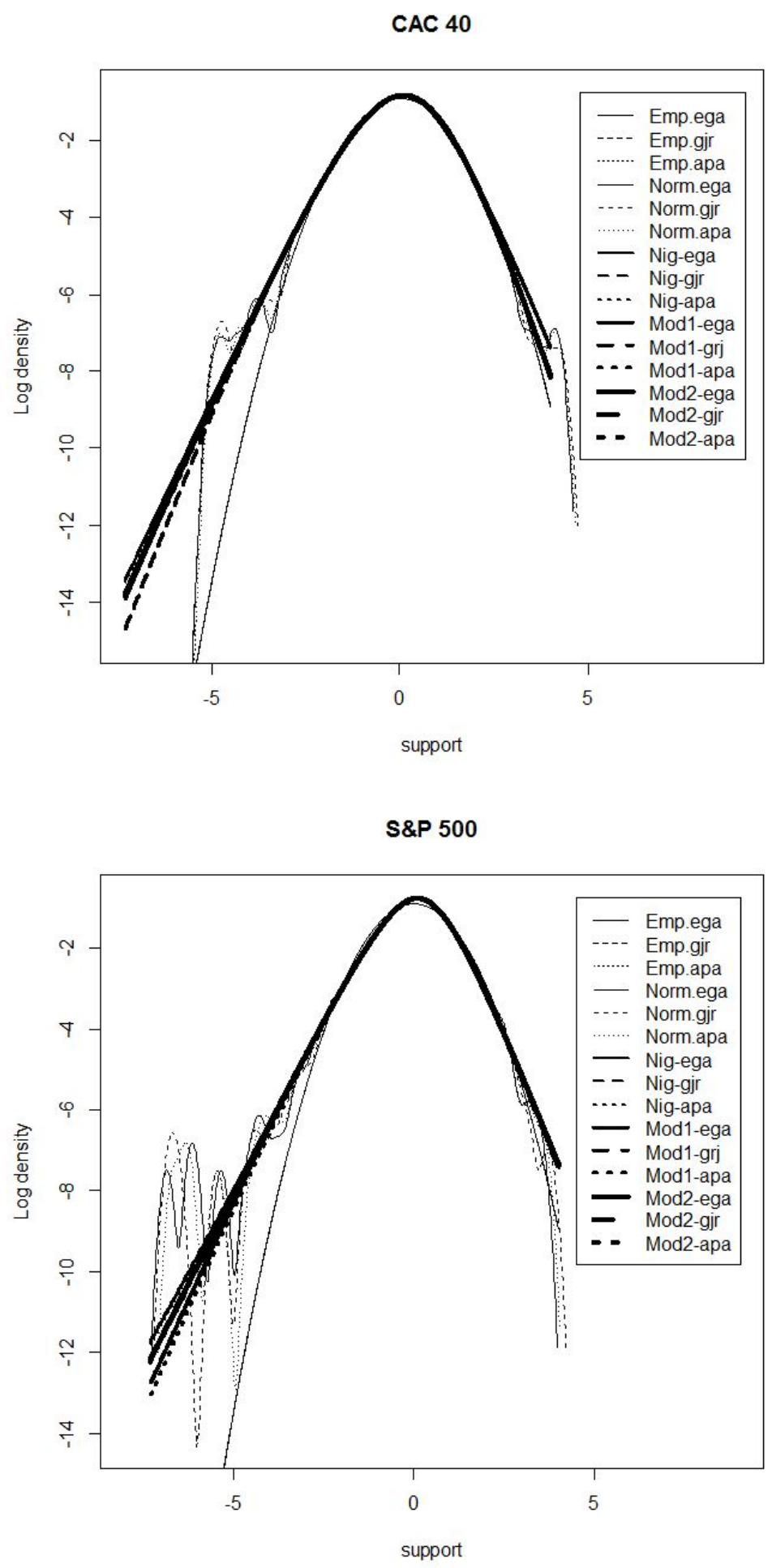

Figure 1: Empirical log-density (solid line) vs. estimated log density obtained with the residuals of the models EGARCH, GJR and APARCH respectively with Normal, NIG and Poisson distributions as defined in (1)-(7), using the daily CAC 40 and the S\&P 500 returns data set from March 1, 1990 to March 1, 2010. 

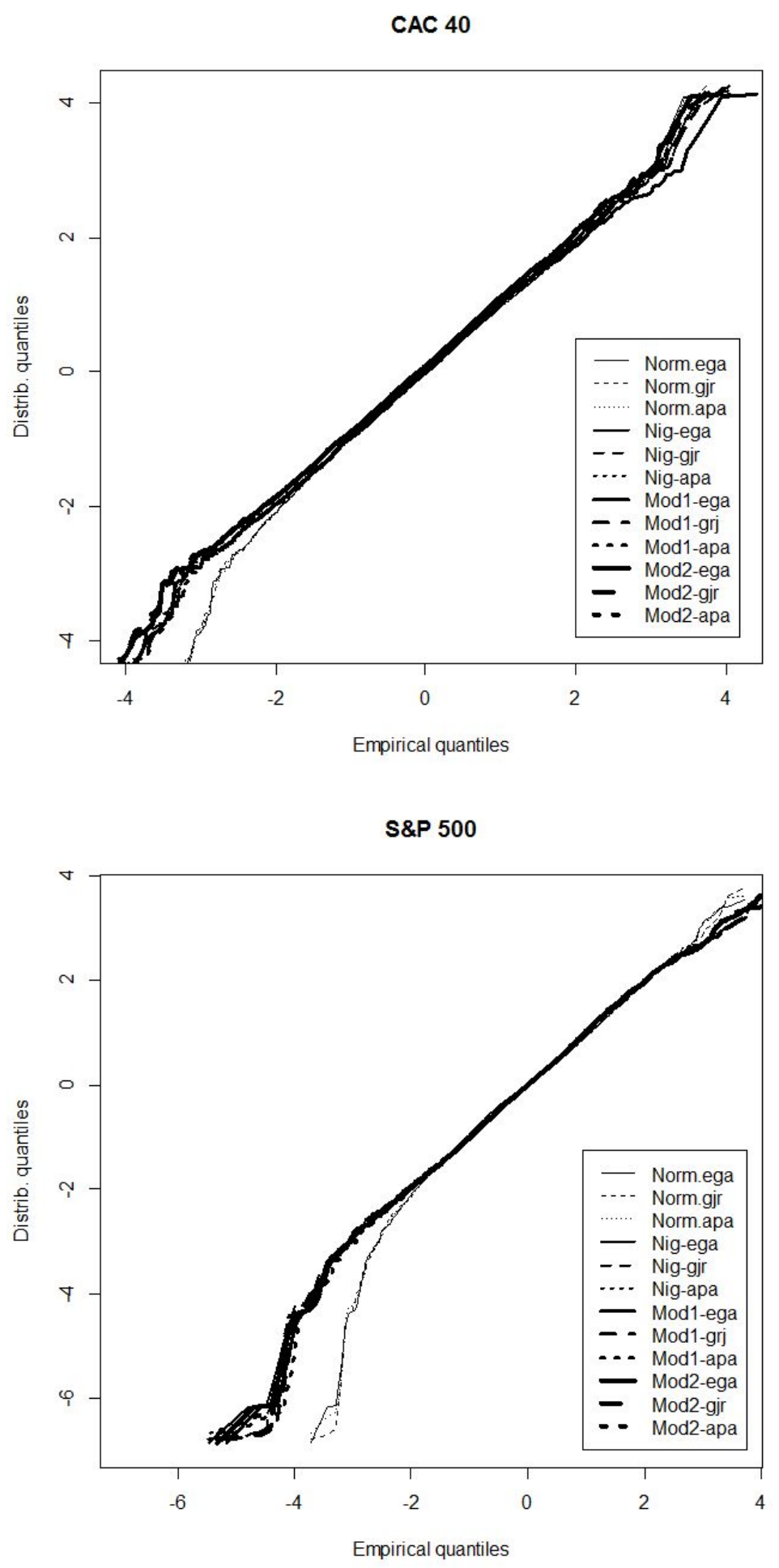

Figure 2: QQ-plots comparing the residuals of the empirical quantiles of the daily CAC 40 and the S\&P $500 \log$ returns vs. the residuals of the estimated quantiles obtained with the models EGARCH, GJR and APARCH respectively with Normal, NIG and Poisson distributions as defined in (1)-(7) for the daily CAC 40 and the S\&P 500 log returns from March 1, 1990 to March 1, 2010. 

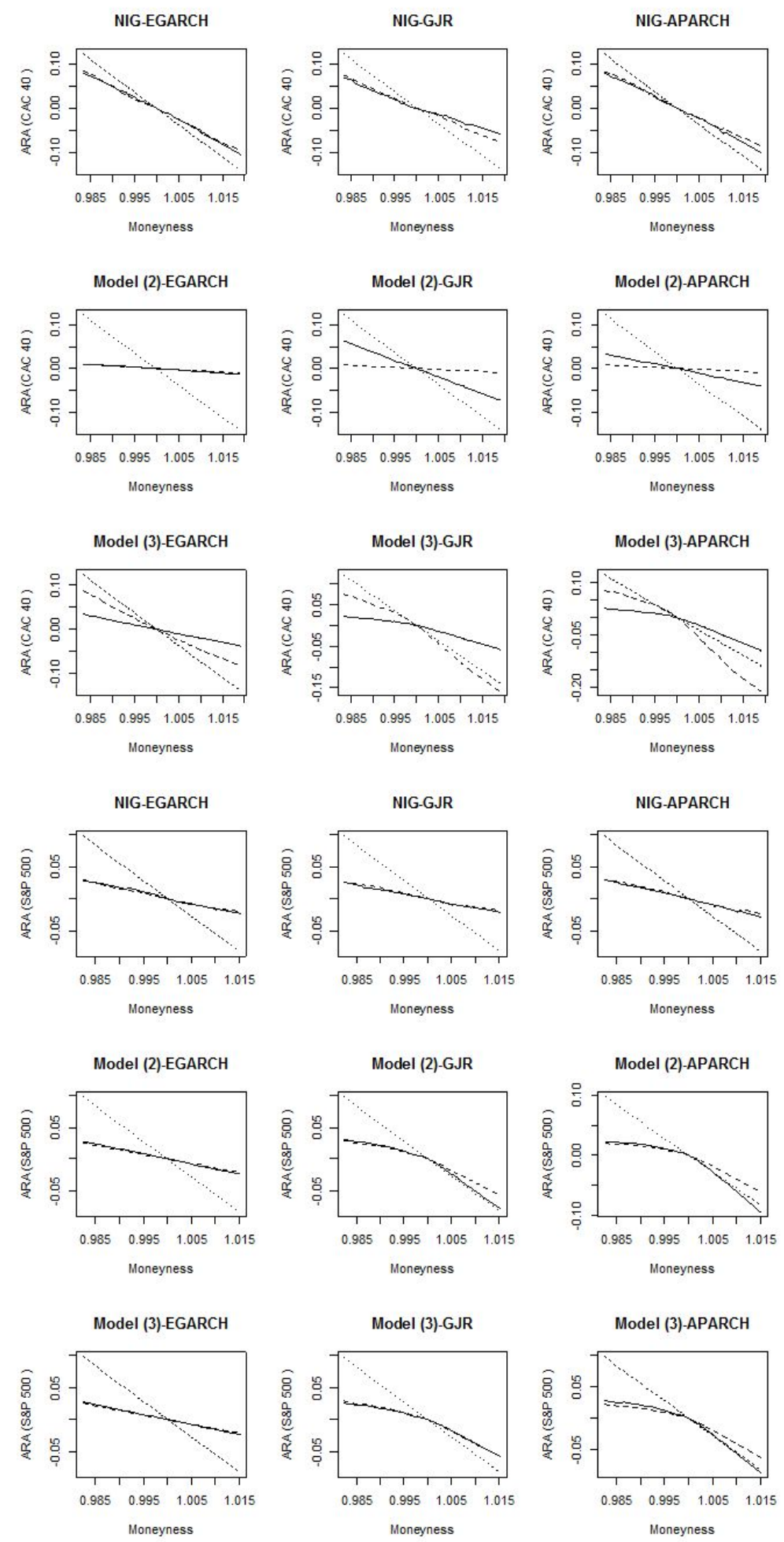

Figure 3: Absolute Risk Aversion (ARA) using the affine pricing kernel (solid lines), the MEMM pricing kernel (dashed) and the ARA of the Black and Scholes model (dotted) of the CAC 40 and the S\&P 500 indexes for 3 months time to maturity, from December 6 , 2006 to February 28, 2007. Each panel corresponds to the three models associated to the three type of GARCH models. 